\title{
A Comparative Study on Sensitivity-Based Damage Detection Methods in Bridges
}

\author{
Akbar Mirzaee, Reza Abbasnia, and Mohsenali Shayanfar \\ Department of Civil Engineering, Iran University of Science and Technology, Narmak, Tehran 16846 13114, Iran \\ Correspondence should be addressed to Reza Abbasnia; abbasnia@iust.ac.ir
}

Received 25 September 2014; Accepted 22 January 2015

Academic Editor: Yaguo Lei

Copyright ( 2015 Akbar Mirzaee et al. This is an open access article distributed under the Creative Commons Attribution License, which permits unrestricted use, distribution, and reproduction in any medium, provided the original work is properly cited.

\begin{abstract}
This paper provides a comparative study on four different sensitivity-based damage detection methods for bridges. The methods investigated in this study are approximation approach, semianalytical discrete approach, and analytical discrete approach, which includes direct differential and adjoint variable methods. These sensitivity-based methods utilize finite element model updating procedure and allow a wide choice of physically meaningful parameters leading to vast range of applications in damage detection. The most important difficulty in these methods is calculation of sensitivity matrix. Calculation of this massive matrix is repeated in each iteration and has a significant effect on the efficiency of method. In this study, the acceleration measurements are simulated from the solution to the forward problem using finite element method under moving load with various speeds, along with the addition of artificially produced measurement noise. Various damaged structures with different damage patterns including single, multiple, and random damage are considered and efficiency of four sensitivity methods is compared. Moreover, various possible sources of error such as the effects of measurement noise as well as initial assumption error in stability of the methods are also discussed.
\end{abstract}

\section{Introduction}

Structural health monitoring is the implementation of a damage identification strategy to different types of structures. SHM is vital to evaluate the fitness of a structure, in various disciplines including aerospace, mechanical and civil engineering, to perform its prescribed tasks properly. The necessity of SHM is highlighted when it is recognized that the performance of structures may change due to a gradual or sudden change in states, load conditions, or response mechanisms.

Bridges are truly the flagships of civil engineering, which attract the greatest attention within the engineering community. This is due to their small safety margins and their great exposure to the public [1].

The main objective of developing the SHM system for bridges is to enhance structural safety. However in bridges, SHM serves other economic benefits such as increased mission reliability, extended life of life-limited components, reduced tests, reduction in "down time," increased equipment reliability, customization of maintenance actions, and greater awareness of operating personnel, resulting in fewer accidents. SHM also promises to help in reducing maintenance costs [2].

SHM is an inverse problem wherein the flaws in the structure are characterized using the measured data for some known inputs [3]. Hence, SHM can be thought of as a system identification problem and classified into two categories, namely, the diagnosis and prognosis. Through diagnosis, one can determine the presence of flaws, their locations, and their extents along with the possibility of looking at the delaying the propagation of flaws in the structure. The prognosis part uses the information of the diagnosis part and determines the remaining life of the structure. Therefore the SHM can be broadly divided into five levels as follows [4]:

level 1: confirming the presence of damage;

level 2: determination of location and orientation of the damage;

level 3: evaluation of the severity of the damage;

level 4: possibility of controlling or delaying the growth of damage; 
level 5: determining the remaining life in the structure (prognosis).

Dynamics-based SHM techniques assess the state of health of a structural component on the basis of the detection and analysis of its dynamic response. Such techniques can be classified on the basis of the type of response being considered for the investigations, on the frequency or time domain of investigation, and on the modality used to excite the component [5].

The developments in the field of structural damage detection (DD) using vibration data of civil engineering structures have been recently studied by several authors; some of them are briefly described herein.

Doebling et al. [6, 7] have presented comprehensive review of literature mainly focusing on frequency-domain methods for damage detection in linear structures and declared that sufficient evidence exists to promote the use of measured vibration data for the detection of damage in structures, using both forced-response testing and long-term monitoring of ambient signals and there is a significant need in this field for research on the integration of theoretical algorithms with application-specific knowledge bases and practical experimental constraints. Another discussion on methods of damage detection and location using natural frequency changes has been presented by Salawu [8] and his study showed that damage detection using vibration frequencies is not very reliable. Zou et al. [9] summarized the methods on vibration-based damage detection and health monitoring for composite structures, especially in delamination modeling techniques and delamination detection.

Alampalli and $\mathrm{Fu}$ [10] and Alampalli et al. [11] conducted laboratory and field studies on bridge structures to investigate the feasibility of measuring bridge vibration for inspection and evaluation. These studies focused on sensitivity of measured modal parameters to damage. Cross diagnosis using multiple signatures involving natural frequencies, mode shapes, modal assurance criteria, and coordinate modal assurance criteria was shown to be necessary to detect the damage. Casas and Aparicio [12] studied concrete bridge structures and investigated dynamic response as an inspection tool to assess bearing conditions and girder cracking. Their study showed the need to investigate more than one natural frequency and also to determine mode shapes in order that the damage could be successfully detected and located.

Damage detection usually requires a mathematical model on the structure in conjunction with experimental modal parameters of the structure. The identification approaches are mainly based on the change in the natural frequencies [13-15], mode shapes [16-18], or measured modal flexibility [7, 19-21].

The frequency-domain DD algorithms have been more widely developed and applied as the amount of measured data is reduced dramatically after the transform; thus they can be handled easily. Unfortunately, the effects of local damage on the natural frequencies and mode shapes of higher modes are greater than lower ones, but they are usually difficult to measure from experiments. In addition, structural damping properties cannot be identified in frequency domain DD.
The time-domain DD may be an attractive one to overcome the drawbacks of the frequency-domain DD. For timedomain DD, the forced vibration responses of the structure are needed in the identification. However, in some cases it is either impractical or impossible to use artificial inputs to excite the civil engineering structures, so natural excitation must be measured along with the structural responses to assess the dynamic characteristics [22, 23]. In recent years, some researchers have investigated both the problem of load identification (moving load and impact load) and modal parameters identification under operational conditions [24, 25]. In addition, identification of the structural parameters applying a moving load has been considered in many papers. Law et al. [26] presented a novel moving force and prestress identification method based on the finite element and the wavelet-based methods for a bridge-vehicle system. Jiang et al. [27] identified the parameter of a vehicle moving on multispan continuous bridges. Zhu and Law [28] presented a method for damage detection of a simply supported concrete bridge structure in time domain using the interaction forces from the moving vehicles as excitation.

Sensitivity-based methods allow a wide choice of physically meaningful parameters and this advantage has led to their widespread use in damage detection. Calculation of sensitivity matrix has a significant effect on the efficiency of these methods. Despite the high importance of calculation method of sensitivity matrix and optimizing its performance in $\mathrm{DD}$ procedure, there is not literature on this regard. In this paper, computational methods for sensitivity matrix are discussed and a novel sensitivity base damage detection method in time-domain referred to as "adjoint variable method" is introduced. Fundamental principles of proposed method are presented and its performance is compared with the conventional methods and it is shown that the numerical cost is considerably reduced by using the concept of adjoint variable.

The outline of the work is as follows: inverse problems along with model updating are briefly introduced in Section 2. Different methods for sensitivity analysis along with introduced method (adjoint variable method) are addressed in Section 3. Numerical simulation is presented in Section 4 and comparison studies are presented in Section 5 with studies on the effect of different factors which may affect the accuracy and efficiency of different methods and finally conclusion will be drawn in the last section.

\section{Finite Element Model Updating and Inverse Problem}

A key step in model-based damage identification is the updating of the finite element model of the structure in such a way that the measured responses can be reproduced by the FE model. A general flowchart of this operation is given in Figure 1. The identification procedure presented in this paper is a sensitivity-based model updating routine. Sensitivity coefficients are the derivatives of the system responses with respect to the physical parameters or input excitation force and are needed in the cost function of the flowchart of Figure 1. 


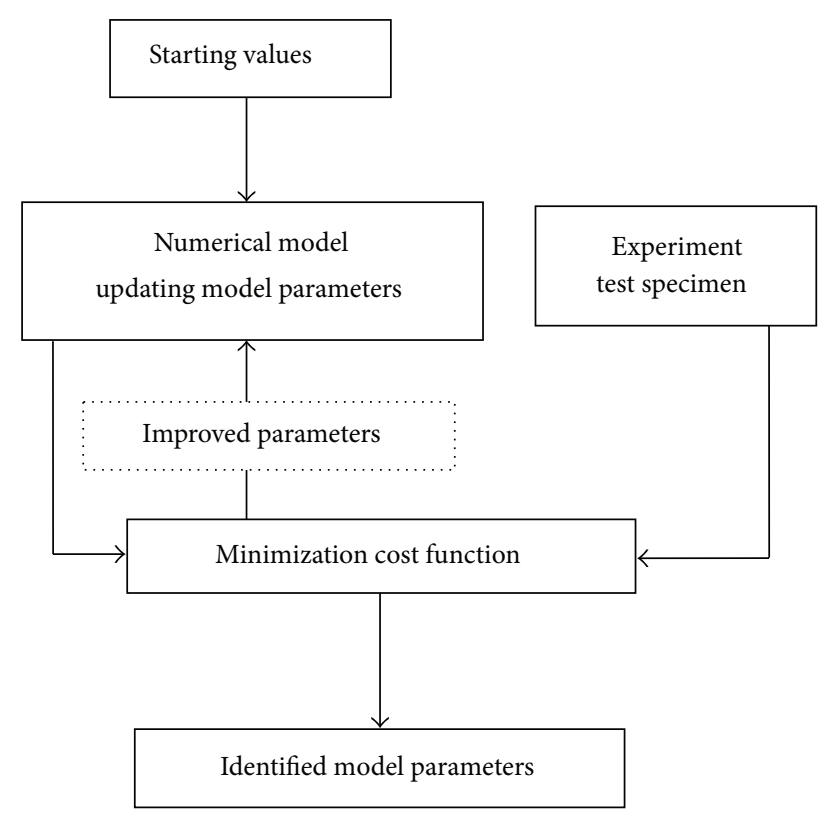

FIGURE 1: General flowchart of a FEM-updating [31].

2.1. Finite Element Modeling of Bridge Vibration under Moving Loads. For a general finite element model of a linear elastic time-invariant structure, the equation of motion is given by

$$
\mathbf{M z} \mathbf{z}_{, t t}+\mathbf{C} \mathbf{z}_{, t}+\mathbf{K z}=\mathbf{B f},
$$

where $\mathbf{M}$ and $\mathbf{K}$ are mass and stiffness matrices and $\mathbf{C}$ is damping matrix. $\mathbf{z}_{t t}$ and $\mathbf{z}_{t}$ and $\mathbf{z}$ are the respective acceleration, velocity, and displacement vectors for the whole structure and $\mathbf{f}$ is a vector of applied forces with matrix $\mathbf{B}$ mapping these forces to the associated DOFs of the structure. Proportional damping is assumed to show the effect of damping ratio on the dynamic magnification factor. Rayleigh damping, in which the damping matrix is proportional to the combination of the mass and stiffness matrices, is used

$$
\mathbf{C}=a_{0} \mathbf{M}+a_{1} \mathbf{K}
$$

where $a_{0}$ and $a_{1}$ are constants to be determined from two modal damping ratios. The dynamic responses of the structures can be obtained by direct numerical integration using Newmark method.

2.2. Objective Functions. The approach minimizes the difference between response quantities (acceleration response) of the measured data and model predictions. This problem may be expressed as the minimization of $j$, where

$$
\begin{array}{r}
j(\theta)=\left\|\mathbf{z}_{m}-\mathbf{z}(\boldsymbol{\alpha})\right\|^{2}=\boldsymbol{\epsilon}^{T} \boldsymbol{\epsilon}, \\
\boldsymbol{\epsilon}=\mathbf{z}_{m}-\mathbf{z}(\boldsymbol{\alpha}) .
\end{array}
$$

Here $\mathbf{z}_{m}$ and $\mathbf{z}(\boldsymbol{\alpha})$ are the measured and computed response vectors, $\boldsymbol{\alpha}$ is a vector of all unknown parameters, and $\boldsymbol{\epsilon}$ is the response residual vector.
2.3. Nonlinear Model Updating for Damage Detection. When the parameters of a model are unknown, they must be estimated using measured data. Since the relationship between the acceleration responses $\ddot{\mathbf{z}}_{i}$ and the fractional stiffness parameter $\boldsymbol{\alpha}$ is nonlinear, a nonlinear model updating technique, like the Gauss-Newton method, is required. This kind of method has the advantage that the second derivatives, which can be challenging to compute, are not required. The Gauss-Newton method in the damage detection procedure can be described in terms of the acceleration response at the $i$ th DOF of the structure as

$$
\begin{aligned}
\ddot{\mathbf{z}}_{d l}\left(\boldsymbol{\alpha}_{d}\right)= & \ddot{\mathbf{z}}_{u l}\left(\boldsymbol{\alpha}^{0}\right)+\mathbf{S}\left(\boldsymbol{\alpha}^{0}\right) \times \Delta \boldsymbol{\alpha}^{1} \\
& +\mathbf{S}\left(\boldsymbol{\alpha}^{0}+\Delta \boldsymbol{\alpha}^{1}\right) \times \Delta \boldsymbol{\alpha}^{2}+\cdots .
\end{aligned}
$$

The superscripts 0,1 , and 2 denote the iteration numbers. Index $u$ denotes the initial state or state 0 while index $d$ denotes the final damage state. $\ddot{\mathbf{z}}_{d l}$ and $\ddot{\mathbf{z}}_{u l}$ are vectors of the acceleration response at the $i$ th DOF of the damaged and intact states, respectively. The damage identification equation for $(k+1)$ th iteration is

$$
\Delta \ddot{\mathbf{z}}^{k}=\mathbf{S}^{k} \times \Delta \boldsymbol{\alpha}^{k+1}
$$

where $\mathbf{S}^{k}$ and $\Delta \ddot{\mathbf{z}}^{k}$ are obtained from the $k$ th iteration. The iteration in (5) starts with an initial value $\boldsymbol{\alpha}^{0}$ leading to $\Delta \ddot{\mathbf{z}}^{0}=$ $\ddot{\mathbf{z}}_{d l}-\ddot{\mathbf{z}}_{u l}\left(\boldsymbol{\alpha}^{0}\right)$ and $\boldsymbol{S}^{0}=\mathbf{S}\left(\boldsymbol{\alpha}^{0}\right)$. The parameter vector $\boldsymbol{\alpha}^{k}=$ $\boldsymbol{\alpha}^{0}+\sum_{i=1}^{k} \Delta \boldsymbol{\alpha}^{i}$. Sensitivity matrix $\mathbf{S}^{k}=\mathbf{S}\left(\boldsymbol{\alpha}^{k}\right)$, and the residual vector $\Delta \ddot{\mathbf{z}}^{k}=\ddot{\mathbf{z}}_{d l}-\ddot{\mathbf{z}}_{u l}\left(\boldsymbol{\alpha}^{0}\right)-\sum_{i=0}^{k-1} \mathbf{S}^{i} \Delta \boldsymbol{\alpha}^{i+1},(k=1,2, \ldots)$ of the next iteration is then computed from results in the previous iterations.

The acceleration response vector $\ddot{\mathbf{z}}_{u l}$ from the physical intact structure is computed, in general, from the associated analytical model via dynamic analysis. $\ddot{\mathbf{z}}_{d l}$ is the acceleration response of the model of the damaged structure. In general, the measured acceleration responses (including measurement errors) from the damaged structure are obtained for $\ddot{\mathbf{z}}_{d l}$.

The iteration is terminated when a preselected criterion is met. The final identified damaged vector becomes [17]

$$
\Delta \boldsymbol{\alpha}=\Delta \boldsymbol{\alpha}^{1}+\Delta \boldsymbol{\alpha}^{2}+\cdots+\Delta \boldsymbol{\alpha}^{n}
$$

where $n$ is the number of iterations.

2.4. Regularization. Like many other inverse problems, the solution of (5) is often ill-conditioned and regularization techniques are needed to provide bounds to the solution. The aim of regularization in the inverse analysis is to promote certain regions of parameter space where the model realization should exist. The two most widely used regularization methods are Tikhonov regularization [14] and truncated singular value decomposition [29]. In the Tikhonov regularization, the new cost function is defined as

$$
j\left(\Delta \boldsymbol{\alpha}^{k+1}, \lambda\right)=\left\|\boldsymbol{S}^{k} \cdot \Delta \boldsymbol{\alpha}^{k+1}-\Delta \ddot{\mathbf{z}}^{k}\right\|_{2}^{2}+\lambda^{2}\left\|\Delta \boldsymbol{\alpha}^{k+1}\right\|_{2}^{2} .
$$

The regularization parameter $\lambda \geq 0$ controls the extent of contribution of the two errors to the cost function in (7) and 
the fractional stiffness change increment $\Delta \boldsymbol{\alpha}^{k+1}$ is obtained by minimizing the cost function in (7).

The regularized solution from minimizing the function in (7) can be written in the following form as

$$
\Delta \boldsymbol{\alpha}^{k+1}=\left(\left(\mathbf{S}^{k}\right)^{T} \mathbf{S}^{k}+\lambda^{2} \mathbf{I}\right)^{-1}\left(\mathbf{S}^{k}\right)^{T} \Delta \ddot{\mathbf{z}}^{k}
$$

To express the contribution of the singular values and the corresponding vectors in the solution clearly and to show how the regularization parameter plays the role as the filter factor, the sensitivity matrix is singular value decomposed and singular value decomposition (SVD) applies to the sensitivity matrix $\mathbf{S}^{k}$ to obtain

$$
\mathbf{S}^{k}=\mathbf{U} \mathbf{\Sigma} \mathbf{V}^{T}
$$

where $\mathbf{U} \in R^{n+\times n t}$ and $\mathbf{V} \in R^{m \times m}$ are orthogonal matrices satisfying $\mathbf{U}^{T} \mathbf{U}=\mathbf{I}_{n t}$ and $\mathbf{V}^{T} \mathbf{V}=\mathbf{I}_{m}$, and matrix $\boldsymbol{\Sigma}$ has the size of $n t \times m$ with the singular values $\sigma_{i}(i=1,2, \ldots, m)$ on the diagonal arranged in a decreasing order such that $\sigma_{1} \geq$ $\sigma_{2} \geq \cdots \geq \sigma_{m} \geq 0$ and zeros elsewhere.

The regularized solution in (8) can be written as

$$
\Delta \boldsymbol{\alpha}^{k+1}=\sum_{i=1}^{m} f_{i} \frac{\mathbf{U}_{i}^{T} \Delta \ddot{\mathbf{z}}^{k}}{\sigma_{i}} \mathbf{V}_{i}
$$

where $f_{i}=\sigma_{i}^{2} /\left(\sigma_{i}^{2}+\lambda^{2}\right)(i=1,2, \ldots, m)$ are referred as filter factors. The solution norm $\left\|\Delta \boldsymbol{\alpha}^{k+1}\right\|_{2}^{2}$ and the residual norm $\left\|\mathbf{S}^{k} \cdot \Delta \boldsymbol{\alpha}^{k+1}-\Delta \ddot{\mathbf{z}}^{k}\right\|_{2}^{2}$ can be expressed as

$$
\begin{gathered}
\eta^{2}=\left\|\Delta \boldsymbol{\alpha}^{k+1}\right\|_{2}^{2}=\sum_{i=1}^{m}\left(\frac{\sigma_{i}^{2}}{\sigma_{i}^{2}+\lambda^{2}} \frac{\mathbf{U}_{i}^{T} \Delta \ddot{\mathbf{z}}^{k}}{\sigma_{i}}\right)^{2}, \\
\rho^{2}=\left\|\mathbf{S}^{k} \cdot \Delta \boldsymbol{\alpha}^{k+1}-\Delta \ddot{\mathbf{z}}^{k}\right\|_{2}^{2}=\sum_{i=1}^{m}\left(\frac{\lambda^{2}}{\sigma_{i}^{2}+\lambda^{2}} \mathbf{U}_{i}^{T} \Delta \ddot{\mathbf{z}}^{k}\right)^{2} .
\end{gathered}
$$

These two quantities represent the smoothness and goodnessof-fit of the solution and they should be balanced by choosing an appropriate regularization parameter.

2.5. Element Damage Index. In the inverse problem of damage identification, it is assumed that the stiffness matrix of the whole element decreases uniformly with damage, and the flexural rigidity $\mathrm{EI}_{i}$ of the $i$ th finite element of the beam becomes $\beta_{i} \mathrm{EI}_{i}$, when there is damage. The fractional change in stiffness of an element can be expressed as [30]

$$
\Delta \mathbf{K}_{b i}=\left(\mathbf{K}_{b i}-\widetilde{\mathbf{K}}_{b i}\right)=\left(1-\beta_{i}\right) \mathbf{K}_{b i},
$$

where $\mathbf{K}_{b i}$ and $\widetilde{\mathbf{K}}_{b i}$ are the $i$ th element stiffness matrices of the undamaged and damaged beam, respectively. $\Delta \mathbf{K}_{b i}$ is the stiffness reduction of the element. A positive value of $\beta_{i} \epsilon$ $[0,1]$ will indicate a loss in the element stiffness. The $i$ th element is undamaged when $\beta_{i}=1$ and the stiffness of the $i$ th element is completely lost when $\beta_{i}=0$.
The stiffness matrix of the damaged structure is the assemblage of the entire element stiffness matrix $\widetilde{\mathbf{K}}_{b i}$

$$
\mathbf{K}_{b}=\sum_{i=1}^{N} \mathbf{A}_{i}^{T} \widetilde{\mathbf{K}}_{b i} \mathbf{A}_{i}=\sum_{i=1}^{N} \beta_{i} \mathbf{A}_{i}^{T} \mathbf{K}_{b i} \mathbf{A}_{i}
$$

where $\mathbf{A}_{i}$ is the extended matrix of element nodal displacement that facilitates assembling of global stiffness matrix from the constituent element stiffness matrix.

\section{Sensitivity Analysis of Transient Dynamic Response}

The objective of sensitivity analysis is to quantify the effects of parameter variations on calculated results. Terms such as influence, importance, ranking by importance, and dominance are all related to sensitivity analysis.

The simplest and most common procedure for assessing the effects of parameter variations on a model is to vary selected input parameters, rerun the code, and record the corresponding changes in the results or responses, calculated by the code. The model parameters responsible for the largest relative changes in a response are then considered to be the most important for the respective response. For complex models, though, the large amount of computing time needed by such recalculations severely restricts the scope of this sensitivity analysis method; in practice, therefore, the modeler who uses this method can investigate only a few parameters that he judges a priori to be important [32].

When the parameter variations are small, the traditional way to assess their effects on calculated responses is by using perturbation theory, either directly or indirectly, via variational principles. The basic aim of perturbation theory is to predict the effects of small parameter variations without actually calculating the perturbed configuration but rather by using solely unperturbed quantities.

3.1. Methods of Structural Sensitivity Analysis. Various methods employed in design sensitivity analysis are listed in Figure 2. Three approaches are used to obtain the design sensitivity: the approximation, discrete, and continuum approaches. In the approximation approach, design sensitivity is obtained by either the forward finite difference or the central finite difference method. In the discrete method, design sensitivity is obtained by taking design derivatives of the discrete governing equation. For this process, it is necessary to take the design derivative of the stiffness matrix. If this derivative is obtained analytically using the explicit expression of the stiffness matrix with respect to the design variable, it is an analytical method, since the analytical expressions of stiffness matrix are used. However, if the derivative is obtained using a finite difference method, the method is called a semianalytical method.

In the continuum approach, the design derivative of the variational equation is taken before it is discretized. If the structural problem and sensitivity equations are solved as a continuum problem, then it is called the continuumcontinuum method. However, only very simple, classical 


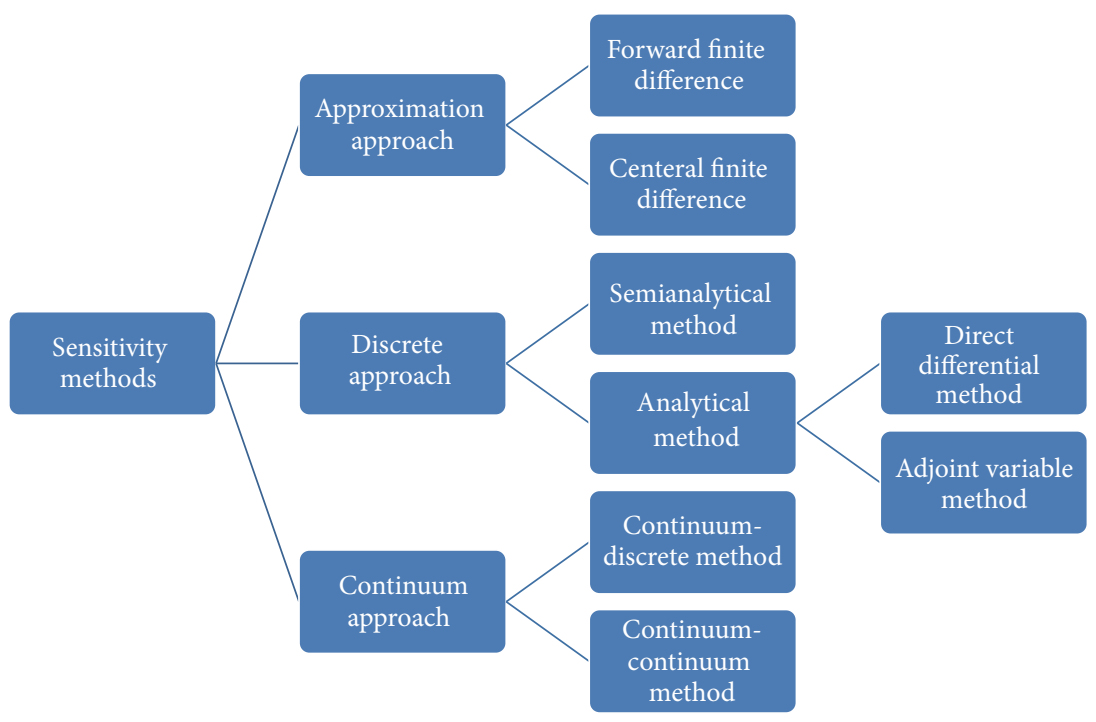

FIGURE 2: Approaches to design sensitivity analysis.

problems can be solved analytically. Thus, the continuum sensitivity equation is solved by discretization in the same way that structural problems are solved. Since differentiation is taken at the continuum domain and is then followed by discretization, this method is called the continuum-discrete method.

3.2. Approximation Approach. The easiest way to compute sensitivity information of the performance measure is by using the finite difference method. Different designs yield different analysis results and, thus, different performance values. The finite difference method actually computes design sensitivity of performance by evaluating performance measures at different stages in the design process. If $\mathbf{u}$ is the current design, then the analysis results provide the value of performance measure $\psi(\mathbf{u})$. In addition, if the design is perturbed to $\mathbf{u}+\Delta \mathbf{u}$, where $\Delta \mathbf{u}$ represents a small change in the design, then the sensitivity of $\psi(\mathbf{u})$ can be approximated as

$$
\frac{d \psi}{d \mathbf{u}} \approx \frac{\psi(\mathbf{u}+\Delta \mathbf{u})-\psi(\mathbf{u})}{\Delta \mathbf{u}}
$$

Equation (14) is called the forward difference method since the design is perturbed in the direction of $+\Delta \mathbf{u}$. If $-\Delta \mathbf{u}$ is substituted in (14) for $\Delta \mathbf{u}$ then the equation is defined as the backward difference method. Additionally, in central difference method, the design is perturbed in both directions and the design sensitivity is approximated by:

$$
\frac{d \psi}{d \mathbf{u}} \approx \frac{\psi(\mathbf{u}+\Delta \mathbf{u})-\psi(\mathbf{u}-\Delta \mathbf{u})}{2 \Delta \mathbf{u}} .
$$

Then the equation is defined as the central difference method.

3.3. Discrete Approach. A structural problem is often discretized in finite dimensional space in order to solve complex problems. The discrete method computes the performance design sensitivity of the discretized problem.

The design represents a structural parameter that can affect the results of the analysis.

The design sensitivity information of a general performance measure can be computed either with the direct differentiation method or with the adjoint variable method.

3.3.1. Direct Differentiation Method. The direct differentiation method (DDM) is a general, accurate, and efficient method to compute FE response sensitivities to the model parameters. This method directly solves for the design dependency of a state variable and then computes performance sensitivity using the chain rule of differentiation. This method clearly shows the implicit dependence on the design, and a very simple sensitivity expression can be obtained.

Consider a structure in which the generalized stiffness and mass matrices have been reduced by accounting for boundary conditions. Let the damping force be represented in the form of $\mathbf{C}(b) \mathbf{z}_{t}$ where $\mathbf{z}_{t}=d \mathbf{z} / d t$ denotes the velocity vector. Under these conditions, Lagrange's equation of motion becomes the second-order differential equation, as [33]

$$
\mathbf{M}(b) \mathbf{z}_{, t t}+\mathbf{C}(b) \mathbf{z}_{, t}+\mathbf{K}(b) \mathbf{z}=\mathbf{f}(t, b) .
$$

With the initial conditions

$$
\mathbf{z}(0)=\mathbf{z}^{0}, \quad \mathbf{z}_{, t}(0)=\mathbf{z}_{, t}^{0} .
$$

If design parameters are just related to stiffness matrix, we obtain

$$
\mathbf{M} \frac{\partial \mathbf{z}_{, t t}}{\partial b^{i}}+\mathbf{C} \frac{\partial \mathbf{z}_{, t}}{\partial b^{i}}+\mathbf{K} \frac{\partial \mathbf{z}}{\partial b^{i}}=-\frac{\partial \mathbf{K}}{\partial b^{i}} \mathbf{z}-\alpha_{2} \frac{\partial \mathbf{K}}{\partial b^{i}} \mathbf{z}_{, t}
$$


in which $\partial \mathbf{z} / \partial \mathbf{b}^{i}, \partial \mathbf{z}_{, t} / \partial \mathbf{b}^{i}$, and $\partial \mathbf{z}_{, t} / \partial \mathbf{b}^{i}$ are sensitivity vectors of displacement, velocity, and acceleration with respect to design parameter $b_{i}$, respectively. Assume that

$$
\begin{gathered}
\mathbf{y}_{, t t}=\frac{\partial \mathbf{z}_{, t t}}{\partial b^{i}}, \\
\mathbf{y}_{, t}=\frac{\partial \mathbf{z}_{, t}}{\partial b^{i}}, \\
\mathbf{y}=\frac{\partial \mathbf{z}}{\partial b^{i}} .
\end{gathered}
$$

So, by replacing (19a), (19b), and (19c) into (18) we have

$$
\mathbf{M y}_{, t t}+\mathbf{C} \mathbf{y}_{, t}+\mathbf{K y}=-\frac{\partial \mathbf{K}}{\partial b^{i}} \mathbf{z}-\alpha_{2} \frac{\partial \mathbf{K}}{\partial b^{i}} \mathbf{z}_{, t}
$$

Right side of (20) can be considered as an equivalent force, so (20) is similar to (16) and sensitivity vectors can be obtained by Newmark method.

3.3.2. Adjoint Variable Method. Mirzaee et al. [34] proved that sensitivity analysis can be performed very efficiently by using deterministic methods based on adjoint functions. This method constructs an adjoint problem that solves for the adjoint variable, which contains all implicitly dependent terms.

The adjoint variable method yields a terminal-value problem, compared with the initial-value problem of response analysis.

For the dynamic structure, the following form of a general performance measure can be considered:

$$
\psi=g(\mathbf{z}(T), b)+\int_{0}^{T} G(\mathbf{z}, b) d t .
$$

General form of sensitivity measure relative to the design parameter $(b)$ is as follows [33]:

$$
\begin{aligned}
\psi^{\prime}= & {\left[\frac{\partial g}{\partial \mathbf{z}}-\frac{\partial g}{\partial \mathbf{z}} \mathbf{z}_{, t}(T)+G(\mathbf{z}(T), b) \frac{1}{\Omega_{, t}} \frac{\partial \Omega}{\partial z}\right] \mathbf{z}^{\prime}(T) } \\
& -\left[\frac{\partial g}{\partial z} \mathbf{z}_{, t}(T)+G(\mathbf{z}(T), b)\right] \frac{1}{\Omega_{, t}} \frac{\partial \Omega}{\partial z_{, t}} \mathbf{z}_{, t}^{\prime}(T) \\
& +\int_{0}^{T}\left[\frac{\partial G}{\partial \mathbf{z}} \mathbf{z}^{\prime}+\frac{\partial G}{\partial b} \delta b\right] d t+\frac{\partial g}{\partial b} \delta b \\
& -\left[\frac{\partial g}{\partial z} \mathbf{z}_{, t}(T)+G(z(T), b)\right] \frac{1}{\Omega_{, t}} \frac{\partial \Omega}{\partial b} \delta b
\end{aligned}
$$

Note that $\psi^{\prime}$ depends on $\mathbf{z}^{\prime}$ and $\mathbf{z}_{, t}^{\prime}$ at $T$, as well as on $\mathbf{z}^{\prime}$ within the integration.

Mirzaee et el. [34] showed that while structural vibration responses are used for damage detection, solving (22) in known time $T$ is as follows:

$$
\begin{gathered}
\frac{d \boldsymbol{\psi}}{d b}(T)=\int_{0}^{T}\left(\widetilde{-\lambda^{T}} a_{0} \frac{\partial \mathbf{K}}{\partial b} \widetilde{\mathbf{Z}_{, t}}-\widetilde{\lambda^{T}} \frac{\partial \mathbf{K}}{\partial b} \widetilde{\mathbf{z}}\right) d t, \\
\lambda_{T}(t)=\mathbf{P}_{T} f(t)+\mathbf{Q}_{T} g(t),
\end{gathered}
$$

in which

$$
\begin{gathered}
\mathbf{P}_{T}=e^{-\xi \omega T} \frac{\lambda_{, t}(T)}{\omega_{D}} \cos \left(\omega_{D} T\right), \\
f(t)=e^{\xi \omega t} \sin \left(\omega_{D} t\right), \\
\mathbf{Q}_{T}=-e^{-\xi \omega T} \frac{\lambda_{, t}(T)}{\omega_{D}} \sin \left(\omega_{D} T\right), \\
g(t)=e^{\xi \omega t} \cos \left(\omega_{D} t\right) .
\end{gathered}
$$

It is worth noting that terminal values for different values of $T$ are not similar and adjoint equation should be calculated for corresponding $T$, separately.

Mirzaee et al. [34] presented a quite efficient incremental solution for solving (24) which significantly improves the proposed method.

3.4. Sensitivity Method Selection. In this paper four different methods are presented to compute sensitivity information of the performance measure for damage detection procedure. First method is the finite Difference method (FD) which is classified as an approximation approach. Second, it is semianalytical discrete method which uses a finite difference scheme to calculate stiffness matrix in direct differential method (DDMFD) and third and fourth methods are two different analytical discrete methods: direct differential method (DDM) and adjoint variable method (ADM).

The first three methods including FD, DDMFD, and DDM are widely used by other authors $[35,36]$ and the last method (ADM) is proposed by the authors (Mirzaee et al. [34]).

3.5. Procedure of Iteration for Damage Detection. The initial analytical model of a structure deviates from the true model and measurement from the initial intact structure is used to update the analytical model. The improved model is then treated as a reference model, and measurement from the damaged structure will be used to update the reference model.

When response measurement from the intact state of the structure is obtained, the sensitivities are computed from presented methods based on the analytical model of the structure and the well knowing input force and velocity. The vector of parameter increments is then obtained using the computed and experimentally obtained responses. The analytical model is then updated and the corresponding response and its sensitivity are again computed for the next iteration. When measurement from the damaged state is obtained, the updated analytical model is used in the iteration in the same way as that using measurement from the intact state. Convergence is considered to be achieved when the following criteria are met:

$$
\begin{gathered}
\frac{\left\|\mathbf{E}_{i+1}-\mathbf{E}_{i}\right\|}{\left\|\mathbf{E}_{i}\right\|} \times 100 \% \leq \text { Tol1 }, \\
\frac{\| \text { Response }_{i+1}-\text { Response }_{i} \|}{\| \text { Response }_{i} \|} \times 100 \% \leq \text { Tol2. }
\end{gathered}
$$


The final vector of identified parameter increments corresponds to the changes occurring in between the two states of the structure. The tolerance is set equal to $1 \times 10^{-5}$ in this study except otherwise specified.

\section{Numerical Results}

In order to evaluate the efficiency and competency of introduced methods, analysis of two FE models with extensive available numerical studies has been carried out. Representative examples are presented to demonstrate the effects of speed of loads, measurement noise level, and initial error on the accuracy and effectiveness of the methods.

The relative percentage error (RPE) in the identified results is calculated from (27), where $\|\cdot\|$ is the norm of matrix and $\mathbf{z}_{, t t_{\text {identified }}}$ and $\mathbf{z}_{, t t_{\text {true }}}$ are the identified and the true acceleration time history, respectively [26];

$$
\mathrm{RPE}=\frac{\left\|\mathbf{z}_{, t t_{\text {identified }}}-\mathbf{z}_{, t t_{\text {true }}}\right\|}{\left\|\mathbf{z}_{, t t_{\text {true }}}\right\|} \times 100 \% .
$$

Since the true value of elastic modulus is unknown, RPE can just be used for evaluating the efficiency of the method.

4.1. Single-Span Bridge. A single-span bridge is studied to compare different methods. This bridge consists of 10 EulerBernoulli beam elements with 11 nodes each with two DOFs. Total length of bridge is $10 \mathrm{~m}$ and height and width of the frame section are $200 \mathrm{~mm}$. Rayleigh damping model is adopted with the damping ratios of the first two modes taken equal to 0.05 .

Structural properties are summarized in Table 1.

The transverse point load $P$ has a constant velocity, $v=$ $L / T$, where $T$ is the traveling time across the bridge and $L$ is the total length of the bridge.

For the forced vibration analysis an implicit time integration method called as "the Newmark integration method" is used with the integration parameters $\beta=1 / 4$ and $\gamma=1 / 2$, which leads to constant-average acceleration approximation.

Speed parameter is defined as

$$
\alpha=\frac{V}{V_{\mathrm{cr}}},
$$

in which $V_{\mathrm{cr}}$ is critical speed $\left(V_{\mathrm{cr}}=(\pi / l) \sqrt{\mathrm{EI} / \rho}\right), V$ is moving load speed, and $\rho$ is mass per unit length of the beams.

4.1.1. Damage Scenarios. Six damage scenarios of single, multiple, and random damage along with initial error in the bridge without measurement noise are studied and shown in Table 2.

Local damage is simulated with a reduction in the elastic modulus of material of an element. The sampling rate is $15000 \mathrm{~Hz}$ and 250 data of the acceleration response (degree of indeterminacy is 25) collected along the $z$-direction at nodes 2 and 7 are used in the identification.

4.1.2. Analysis Results. Tables 3-6 show the results of different detection methods including solution time (ST), number of loops (NL), and RPE. These are the most important parameters to compare the efficiency and accuracy of different methods.

4.1.3. Effect of Noise. Noise is the random fluctuation in the value of measured or input that causes random fluctuation in the output value. Noise at the sensor output is due to either internal noise sources, such as resistors at finite temperatures, or externally generated mechanical and electromagnetic fluctuations [10].

To evaluate the sensitivity of results to such measurement noise, noise-polluted measurements are simulated by adding to the noise-free acceleration vector a corresponding noise vector whose root-mean-square (RMS) value is equal to a certain percentage of the RMS. value of the noise-free data vector. The components of all the noise vectors are of Gaussian distribution, uncorrelated and with a zero mean and unit standard deviation. Then on the basis of the noise-free acceleration $\mathbf{z}_{t, t}$ the noise-polluted acceleration $\mathbf{z}_{, t t_{\mathrm{np}}}$ of the bridge at location $x$ can be simulated by

$$
\mathbf{z}_{, t t_{\mathrm{np}}}=\mathbf{z}_{, t t_{\mathrm{nf}}}+\operatorname{RMS}\left(\mathbf{z}_{, t t_{\mathrm{nf}}}\right) \times N_{\text {level }} \times N_{\text {unit }},
$$

where RMS $\left(\mathbf{z}_{, t t_{\mathrm{nf}}}\right)$ is the RMS value of the noise-free acceleration vector $\mathbf{z}_{, t t_{\mathrm{nf}}} \times N_{\text {level }}$ is the noise level, and $N_{\text {unit }}$ is a randomly generated noise vector with zero mean and unit standard deviation [36].

In order to study effects of noise in stability of different sensitivity methods, scenario 3 (speed ratio of moving load is considered to be fixed and equal to 0.5 ) is considered and different levels of noise pollution are investigated, and also RPE change with increasing the number of loops in iterative procedure has been studied. The results are illustrated in Figure 3 for all presented methods.

4.1.4. Effect of Initial Error. In order to study effects of initial error in stability of sensitivity methods, scenario 6 (speed ratio of moving load is considered to be fixed and equal to 0.5 ) is considered. This scenario consists of no simulated damage in the structure, but the initial elastic modulus of material of all the elements is underestimated and different levels of initial error are investigated; RPE changes with increasing the number of loops in iterative procedure have been studied. The results are illustrated in Figure 4 for all presented methods.

4.2. Plane Grid Model. A plane grid model of bridge is studied as another numerical example to investigate the efficiency and accuracy of discrete methods. It is notable that according to complexity of this model, approximation and semidiscrete methods are not useable and diverged in all scenarios in all speed ranges.

The structure is modeled by 46 frame elements and 32 nodes with three DOFs at each node for the translation and rotational deformations. Rayleigh damping model is adopted with the damping ratios of the first two modes taken equal to 0.05 . The finite element model of the structure is shown in Figure 5 and structural properties are summarized in Table 1 . 


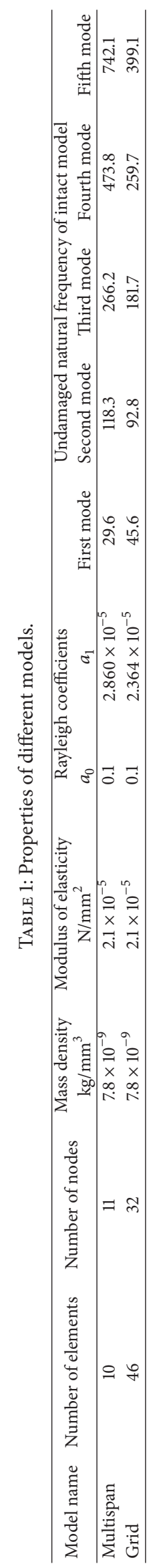


TABLE 2: Damage scenarios for single-span bridge.

\begin{tabular}{|c|c|c|c|c|}
\hline Damage scenario & Damage type & Damage location & Reduction in elastic modulus & Noise \\
\hline M1-1 & Single & 4 & $17 \%$ & Nil \\
\hline M1-2 & Multiple & 2,7 & $4 \%, 21 \%$ & Nil \\
\hline M1-3 & Multiple & $3,5,6$, and 8 & $12 \%, 6 \%, 5 \%$, and $2 \%$ & Nil \\
\hline M1-4 & Random & All elements & Random damage in all elements with an average of $5 \%$ & Nil \\
\hline M1-5 & Random & All elements & Random damage in all elements with an average of $15 \%$ & Nil \\
\hline M1-6 & Estimation of undamaged state & All elements & $5 \%$ reduction in all elements & Nil \\
\hline
\end{tabular}

TABLE 3: Solution time, number of loops, and RPE of ADM method for model 1.

\begin{tabular}{|c|c|c|c|c|c|c|c|c|c|c|c|c|c|c|c|}
\hline \multirow{3}{*}{ Damage scenario } & \multicolumn{15}{|c|}{ Speed parameter } \\
\hline & \multicolumn{3}{|c|}{0.1} & \multicolumn{3}{|c|}{0.3} & \multicolumn{3}{|c|}{0.5} & \multicolumn{3}{|c|}{0.7} & \multicolumn{3}{|c|}{0.9} \\
\hline & $\mathrm{ST}(\mathrm{s})$ & $\mathrm{NL}$ & RPE & ST $(s)$ & NL & $\mathrm{RPE}$ & ST (s) & NL & RPE & ST $(s)$ & NL & RPE & $\mathrm{ST}(\mathrm{s})$ & NL & $\mathrm{RPE}$ \\
\hline M1-1 & 3.658 & 16 & $7.41 E-04$ & 2.719 & 15 & $5.58 E-04$ & 2.742 & 15 & $7.80 E-04$ & 2.953 & 13 & $1.06 E-02$ & 3.049 & 17 & $7.84 E-04$ \\
\hline M1-2 & 4.142 & 16 & $5.92 E-04$ & 3.225 & 14 & $5.35 E-04$ & 2.873 & 15 & $6.91 E-04$ & 2.576 & 14 & $1.02 E-03$ & 3.098 & 17 & $7.00 E-04$ \\
\hline M1-3 & 3.210 & 14 & $8.18 E-04$ & 2.996 & 13 & $8.93 E-04$ & 2.097 & 12 & $5.71 E-04$ & 2.181 & 12 & $9.32 E-04$ & 2.849 & 16 & $5.39 E-04$ \\
\hline M1-4 & 3.966 & 15 & $9.06 E-04$ & 2.744 & 12 & $1.03 E-03$ & 2.548 & 14 & $6.14 E-04$ & 2.821 & 15 & $1.16 E-02$ & 3.078 & 16 & $5.19 E-04$ \\
\hline M1-5 & 3.336 & 15 & $1.02 E-03$ & 2.699 & 12 & $8.31 E-04$ & 2.514 & 14 & $9.07 E-04$ & 3.360 & 17 & $5.16 E-03$ & 2.699 & 15 & $6.61 E-04$ \\
\hline M1-6 & 3.512 & 16 & $5.83 E-04$ & 2.731 & 15 & $5.12 E-04$ & 2.573 & 14 & $7.64 E-04$ & 2.907 & 13 & $1.70 E-03$ & 2.825 & 16 & $6.25 E-04$ \\
\hline
\end{tabular}

TABLE 4: Solution time, loops, and RPE of DDM method for model 1.

\begin{tabular}{|c|c|c|c|c|c|c|c|c|c|c|c|c|c|c|c|}
\hline \multirow{3}{*}{ Damage scenario } & \multicolumn{15}{|c|}{ Speed parameter } \\
\hline & \multicolumn{3}{|c|}{0.1} & \multicolumn{3}{|c|}{0.3} & \multicolumn{3}{|c|}{0.5} & \multicolumn{3}{|c|}{0.7} & \multicolumn{3}{|c|}{0.9} \\
\hline & ST & NL & RPE & ST & NL & RPE & ST & NL & RPE & ST & NL & RPE & ST & NL & RPE \\
\hline M1-1 & 6.947 & 11 & $2.25 E-03$ & 7.081 & 12 & $1.59 E-03$ & 7.171 & 12 & $2.17 E-03$ & 9.711 & 11 & $2.89 E-03$ & 6.532 & 11 & $2.00 E-03$ \\
\hline M1-2 & 11.994 & 13 & $2.44 E-03$ & 13.07 & 13 & $1.61 E-03$ & 7.130 & 12 & $1.59 E-03$ & 8.345 & 14 & $2.35 E-03$ & 7.765 & 13 & $1.68 E-03$ \\
\hline M1-3 & 8.733 & 12 & $2.37 E-03$ & 10.64 & 10 & $1.85 E-03$ & 6.070 & 9 & $1.42 E-03$ & 5.790 & 9 & $2.16 E-03$ & 5.978 & 9 & $3.03 E-03$ \\
\hline M1-4 & 7.071 & 11 & $1.98 E-03$ & 8.892 & 10 & $1.66 E-03$ & 6.531 & 11 & $3.13 E-03$ & 5.938 & 10 & $1.89 E-03$ & 5.347 & 9 & $1.66 E-03$ \\
\hline M1-5 & 9.629 & 15 & $1.73 E-03$ & 10.69 & 12 & $2.18 E-03$ & 7.727 & 13 & $1.98 E-03$ & 5.278 & 9 & $2.05 E-03$ & 7.620 & 13 & $2.88 E-03$ \\
\hline M1-6 & 5.949 & 9 & $2.15 E-03$ & 5.998 & 10 & $1.21 E-03$ & 6.965 & 11 & $2.80 E-03$ & 9.194 & 9 & $8.26 E-04$ & 8.026 & 12 & $1.58 E-03$ \\
\hline
\end{tabular}

TABLE 5: Solution time, loops, and RPE of FD method for model 1.

\begin{tabular}{|c|c|c|c|c|c|c|c|c|c|c|c|c|c|c|c|}
\hline \multirow{3}{*}{ Damage scenario } & \multicolumn{15}{|c|}{ Speed parameter } \\
\hline & \multicolumn{3}{|c|}{0.1} & \multicolumn{3}{|c|}{0.3} & \multicolumn{3}{|c|}{0.5} & \multicolumn{3}{|c|}{0.7} & \multicolumn{3}{|c|}{0.9} \\
\hline & $\mathrm{ST}(\mathrm{s})$ & NL & RPE & ST (s) & NL & RPE & $\mathrm{ST}(\mathrm{s})$ & NL & RPE & $\mathrm{ST}(\mathrm{s})$ & NL & RPE & $\mathrm{ST}(\mathrm{s})$ & NL & RPE \\
\hline M1-1 & 163.8 & 217 & 0.027 & 148.2 & 197 & 0.071 & 225.5 & $300^{*}$ & 0.101 & 257.6 & $300^{*}$ & 0.418 & 226.7 & $300^{*}$ & 0.923 \\
\hline M1-2 & 249.8 & 280 & 0.028 & 167.4 & 196 & 0.048 & 227.3 & $300^{*}$ & 0.189 & 227.1 & $300^{*}$ & 1.322 & 226.9 & $300^{*}$ & 0.627 \\
\hline M1-3 & 158.7 & 208 & 0.199 & 134.7 & 154 & 0.022 & 216.6 & 284 & 0.081 & 187.0 & 244 & 0.134 & 229.2 & $300^{*}$ & 0.570 \\
\hline M1-4 & 97.8 & 114 & 0.127 & 94.1 & 125 & 0.057 & 225.7 & $300^{*}$ & 0.072 & 226.8 & $300^{*}$ & 0.391 & 226.4 & $300^{*}$ & 0.627 \\
\hline M1-5 & 257.7 & $300^{*}$ & 0.030 & 123.0 & 160 & 0.031 & 323.9 & $300^{*}$ & 0.086 & 228.0 & $300^{*}$ & 0.367 & 230.2 & $300^{*}$ & 0.399 \\
\hline M1-6 & 107.6 & 140 & 0.019 & 94.3 & 125 & 0.029 & 225.7 & $300^{*}$ & 0.064 & 282.1 & $300^{*}$ & 0.536 & 228.5 & $300^{*}$ & 0.732 \\
\hline
\end{tabular}

${ }^{*}$ Max number of iterations reached and not converged.

4.2.1. Damage Scenarios. Six damage scenarios of single, multiple, and random damage in the bridge without measurement noise are studied and shown in Table 7.

The sampling rate is $14000 \mathrm{~Hz}$ and 460 data of the acceleration response (degree of indeterminacy is 20) collected along the $z$-direction at nodes $4,11,21$, and 27 are used.

4.2.2. Analysis Results. Tables 8 and 9 show the results of different detection methods including solution time (ST) and RPE. Using both described methods, including ADM and DDM method, the damage locations and amount are identified correctly in all the scenarios (Figure 6).

4.2.3. Effect of Noise. In order to study effects of noise, scenario 3 is considered and different levels of noise pollution are investigated, and also RPE changes with increasing the number of loops for iterative procedure have been studied. The results are presented in Figure 7. 
TABLE 6: Solution time, loops, and RPE of DDMFD method for model 1.

\begin{tabular}{|c|c|c|c|c|c|c|c|c|c|c|c|c|c|c|c|}
\hline \multirow{3}{*}{ Damage scenario } & \multicolumn{15}{|c|}{ Speed parameter } \\
\hline & \multicolumn{3}{|c|}{0.1} & \multicolumn{3}{|c|}{0.3} & \multicolumn{3}{|c|}{0.5} & \multicolumn{3}{|c|}{0.7} & \multicolumn{3}{|c|}{0.9} \\
\hline & ST (s) & NL & RPE & ST (s) & NL & RPE & ST (s) & NL & $\mathrm{RPE}$ & $\mathrm{ST}(\mathrm{s})$ & NL & RPE & ST (s) & NL & RPE \\
\hline M1-1 & 110.7 & 176 & 0.065 & 103.7 & 172 & 0.067 & 151.1 & 250 & 0.118 & 293.1 & $300^{*}$ & 0.243 & 207.1 & $300^{*}$ & 0.830 \\
\hline M1-2 & 180.6 & 206 & 0.079 & 150.8 & 165 & 0.088 & 109.8 & 170 & 0.773 & 203.3 & $300^{*}$ & 0.609 & 214.9 & $300^{*}$ & 0.573 \\
\hline M1-3 & 92.6 & 153 & 0.227 & 111.3 & 123 & 0.096 & 121.1 & 194 & 0.143 & 106.2 & 157 & 0.492 & 200.1 & $300^{*}$ & 0.449 \\
\hline M1-4 & 118.5 & 131 & 0.165 & 87.3 & 101 & 0.078 & 80.9 & 130 & 0.125 & 155.0 & 229 & 0.192 & 201.7 & 294 & 0.242 \\
\hline M1-5 & 100.6 & 167 & 0.107 & 158.2 & 191 & 0.049 & 168.7 & 258 & 0.161 & 181.3 & 267 & 0.254 & 203.5 & $300^{*}$ & 0.670 \\
\hline M1-6 & 70.1 & 101 & 0.253 & 71.5 & 102 & 0.084 & 114.3 & 167 & 0.136 & 229.1 & 223 & 0.275 & 228.6 & $300^{*}$ & 0.257 \\
\hline
\end{tabular}

${ }^{*}$ Max number of iterations reached and not converged.

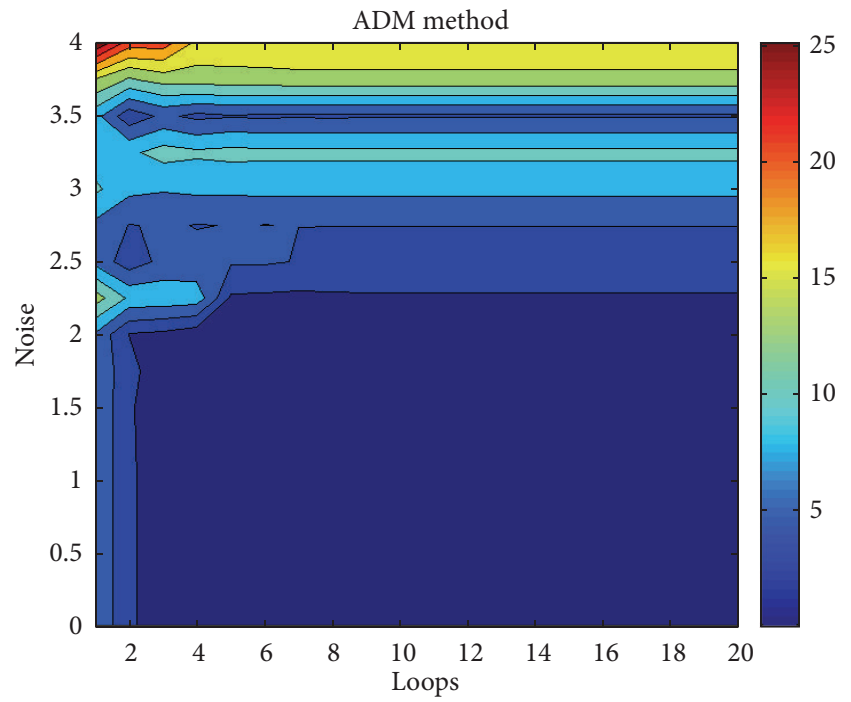

(a) ADM method

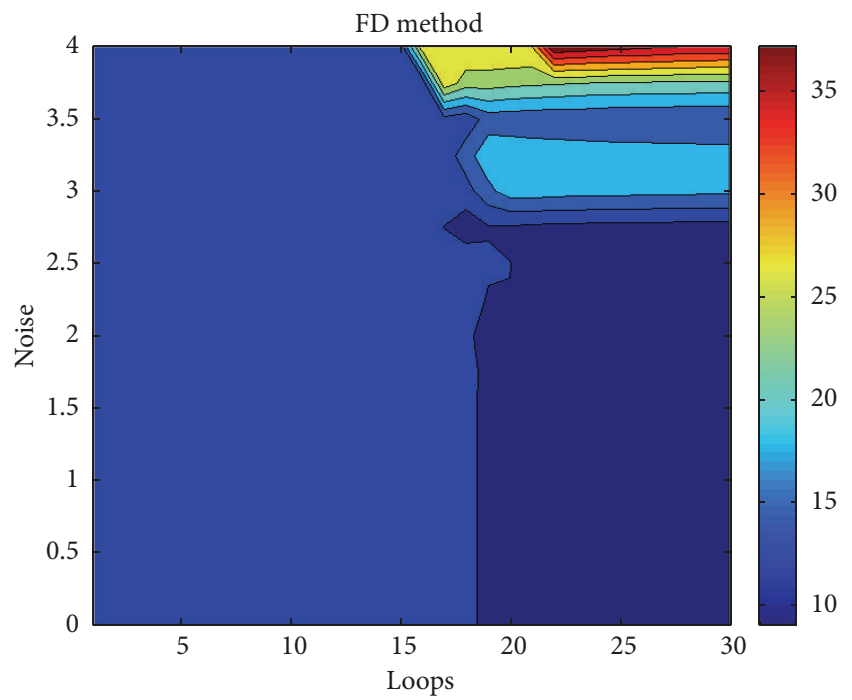

(c) FD method

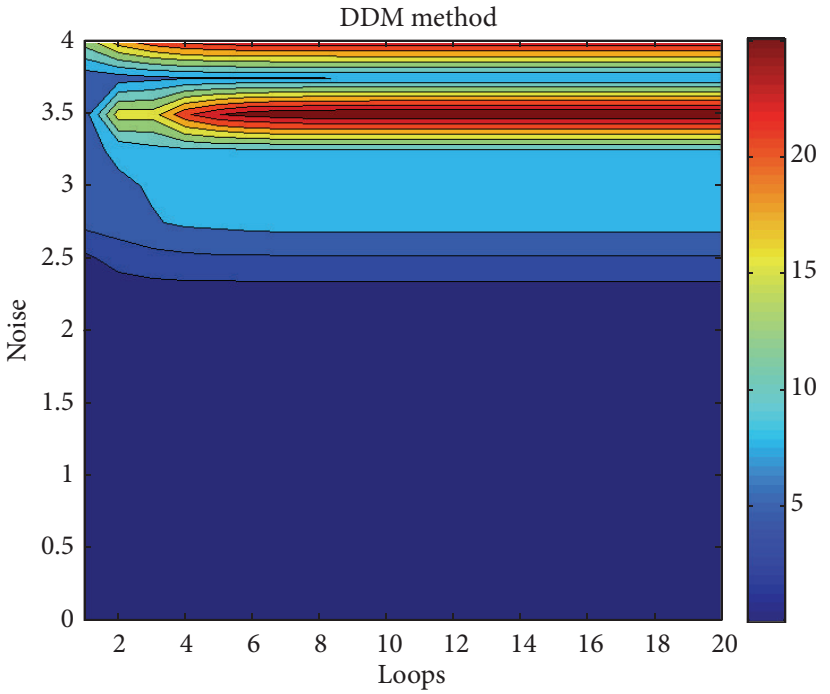

(b) DDM method

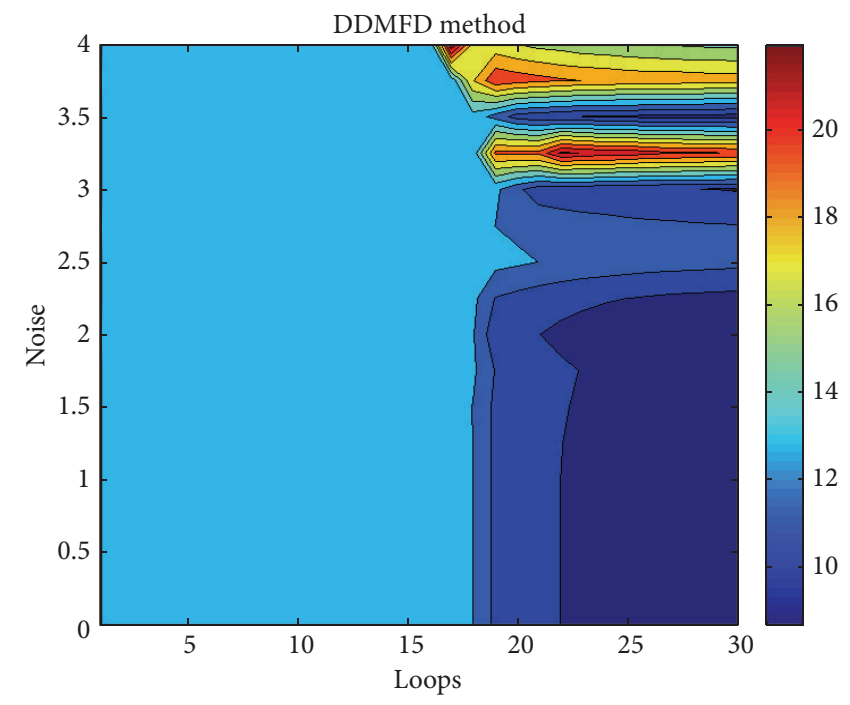

(d) DDMFD method

Figure 3: RPE contours with respect to noise level and loops in model 1. 


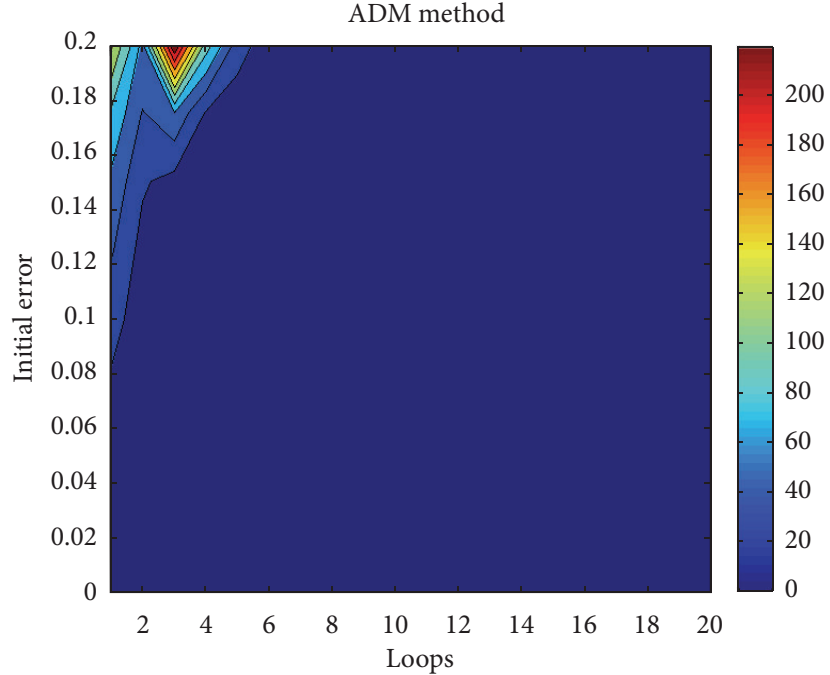

(a) ADM method

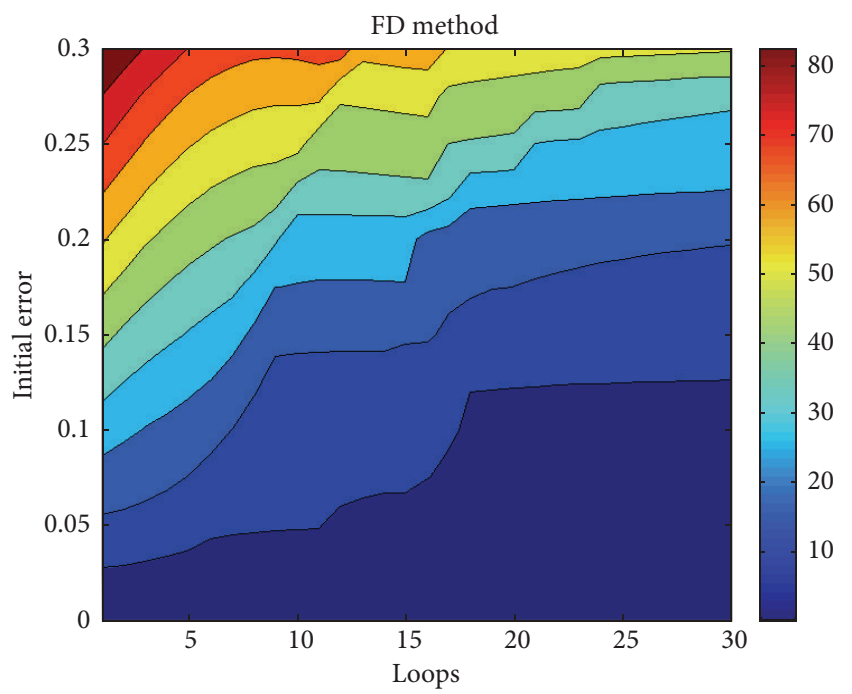

(c) FD method

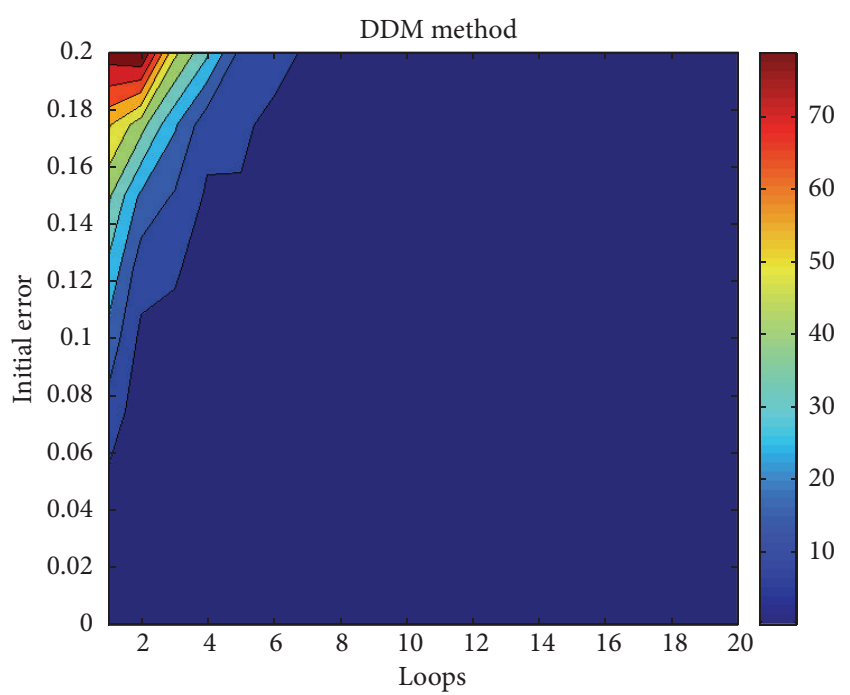

(b) DDM method

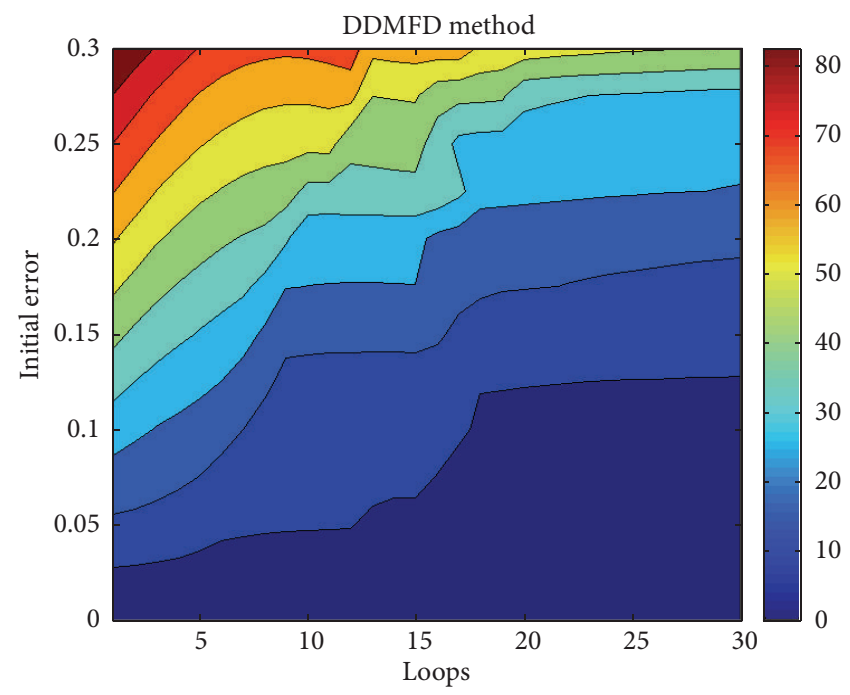

(d) DDMFD method

FIGURE 4: RPE contours with respect to initial error and loops in model 1.

4.2.4. Effect of Initial Error. Scenario 6 (speed ratio of moving load is considered to be fixed and equal to 0.5 ) is considered to study effects of initial error in stability of sensitivity methods. RPE changes with increasing the number of loops for iterative procedure have been studied. The results are illustrated in Figure 8.

\section{Comparison of Different Methods}

According to the results obtained in the previous section, different sensitivity methods considered in this study are compared in this section. Iteration number, relative percentage of error (RPE), solution time, noise level, and initial error effect in stability of damage detection procedure are effective parameters to compare efficiency and accuracy of the methods.

5.1. Iteration Number. Average number of iterations for different scenarios with respect to the speed parameter is illustrated in Figure 9.
As seen in Figure 9(a), the number of iterations in FD and DDMFD methods is considerably larger than those of analytical discrete methods (DDM and ADM). It has been observed that in all cases DDMFD method has fewer iterations than FD method. Average number of iterations is 207.57 and 251.467 for DDMFD and FD methods, respectively. The larger the speed ratio of moving vehicle is the larger the number of iterations in approximation methods is. In FD method, for speed ratios more than 0.5 even by using maximum number of iterations (300) convergence was not achieved, while the ratio used in DDMFD method is 0.9 .

Figure 9(b) shows comparison along with analytical discrete methods. It can be seen that DDM has smaller iteration number in all cases. Average number of iterations is 14.6 and 11.167 for ADM and DDM methods, respectively. Both analytical discrete methods converged in all cases with maximum iterations equal to 17 and increment in speed ratio does not affect the number of iterations. 


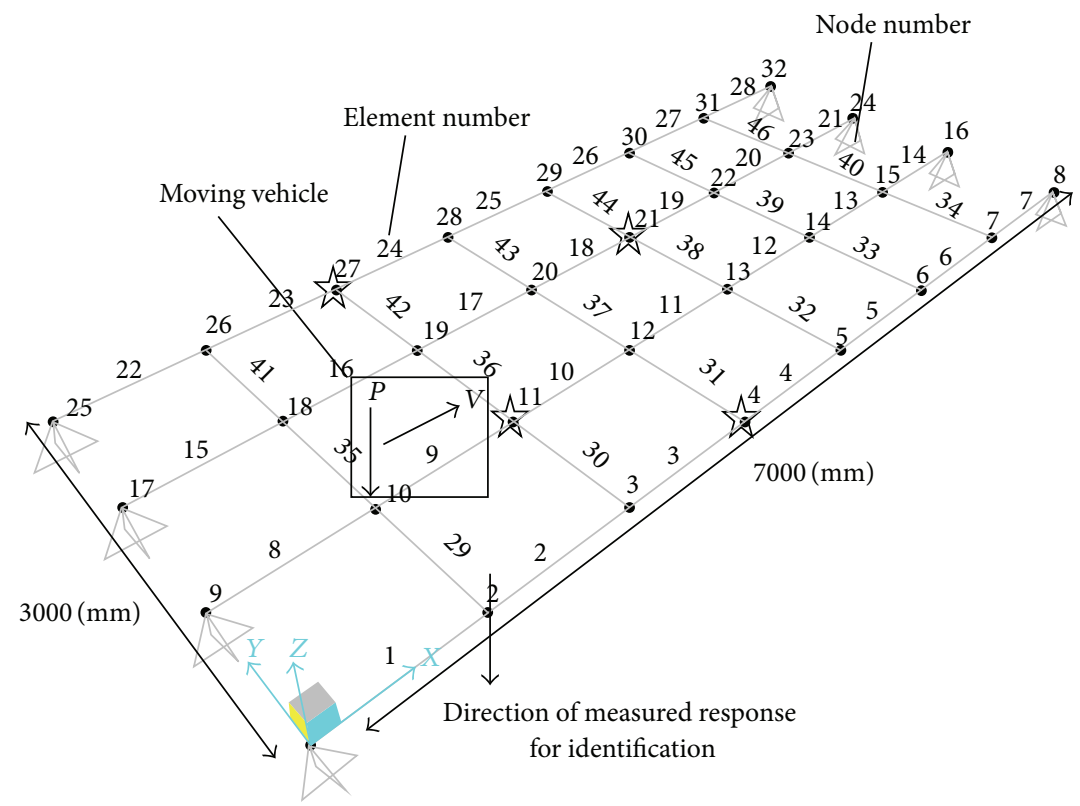

¿ Sensors

FIGURE 5: Plane grid bridge model used in detection procedure.

TABLE 7: Damage scenarios for multispan bridge.

\begin{tabular}{|c|c|c|c|c|}
\hline Damage scenario & Damage type & Damage location & Reduction in elastic modulus & Noise \\
\hline M1-1 & Single & 23 & $5 \%$ & Nil \\
\hline M1-2 & Multiple & 8,13 , and 29 & $11 \%, 4 \%$, and $7 \%$ & Nil \\
\hline M1-3 & Multiple & $3,7,19,25$, and 28 & $12 \%, 6 \%, 5 \%, 2 \%$, and $18 \%$ & Nil \\
\hline M1-4 & Random & All elements & Random damage in all elements with an average of $5 \%$ & Nil \\
\hline M1-5 & Random & All elements & Random damage in all elements with an average of $15 \%$ & Nil \\
\hline M1-6 & Estimation of undamaged state & All elements & $5 \%$ reduction in all elements & Nil \\
\hline
\end{tabular}

5.2. Relative Percentage of Error (RPE). Using the same convergence tolerance equal to $1 \times 10^{-5}$ relative percentage of error in different methods is illustrated in Figure 10.

As seen in this figure, with a maximum number of iterations equal to 300, RPE in FD and DDMFD methods are considerably larger than DDM and ADM methods. DDMFD method has lower RPE than FD method in most cases. Average of RPE is 0.26329 for DDMFD compared to 0.27766 in FD method. Again, the larger the speed ratio of moving vehicle is, the larger the relative error percentage in approximation methods is.

Figure 10(b) shows that relative error percentage in ADM method is lower than DDM and speed ratio does not affect the relative error.

Average of RPE is 0.000731 and 0.002037 for ADM and DDM methods, respectively. Therefore ADM is nearly 2.8 times more accurate than DDM.

5.3. Solution Time. In order to investigate computational cost of sensitivity methods, using the same amounts of RAM and CPU resources for the presented numerical simulations, solution time of different damaged models is evaluated and shown in Figure 11.
As seen in Figure 11(a) solution times in FD and DDMFD methods are considerably larger than analytical discrete methods and the FD method with an average of 200.616 (s) is the most time consuming method. Average time for DDMFD method is 150.849 (s).

Figure 11(b) compares the time spent by DDM and ADM. As illustrated in this figure ADM method has lower solution time than DDM method in all cases and speed ratio does not affect the solution time.

Average of solution time is 2.956 (s) and 7.794 (s) for ADM and DDM methods, respectively; consequently ADM is nearly 2.6 times faster than DDM.

5.4. Efficiency Parameter. In order to compare and quantify the performance of different methods, relative efficiency parameters of methods " $i$ " and " $j$ " $\left(\operatorname{REP}_{i, j}\right)$ are defined as

$$
\mathrm{REP}_{i, j}=\sqrt{\frac{\mathrm{ST}_{j}}{\mathrm{ST}_{i}} \times \frac{\mathrm{RPE}_{j}}{\mathrm{RPE}_{i}}},
$$

in which, ST is the solution time of system identification method that represents the computational cost of the method and RPE is the relative error which represents the accuracy of the method. 
TABLE 8: Solution time, number of loops, and RPE of ADM method for model 2.

\begin{tabular}{|c|c|c|c|c|c|c|c|c|c|c|}
\hline \multirow{3}{*}{ Damage scenario } & \multicolumn{10}{|c|}{ Speed parameter } \\
\hline & \multicolumn{2}{|r|}{0.1} & \multicolumn{2}{|c|}{0.3} & \multicolumn{2}{|c|}{0.5} & \multicolumn{2}{|c|}{0.7} & \multicolumn{2}{|c|}{0.9} \\
\hline & $\mathrm{ST}(\mathrm{s})$ & RPE (\%) & ST (s) & RPE (\%) & $\mathrm{ST}(\mathrm{s})$ & RPE (\%) & ST (s) & RPE (\%) & ST (s) & RPE (\%) \\
\hline M1-1 & 71.527 & $1.26 E-03$ & 75.320 & $2.03 E-03$ & 72.340 & $1.09 E-03$ & 54.745 & $2.30 E-03$ & 75.389 & $1.74 E-03$ \\
\hline M1-2 & 94.756 & $2.15 E-03$ & 95.091 & $2.40 E-03$ & 88.962 & $1.52 E-03$ & 63.255 & $2.05 E-03$ & 93.939 & $1.77 E-03$ \\
\hline M1-3 & 158.37 & $2.62 E-03$ & 106.582 & $1.86 E-03$ & 96.323 & $1.26 E-03$ & 92.704 & $2.12 E-03$ & 166.000 & $1.71 E-03$ \\
\hline M1-4 & 89.368 & $2.43 E-03$ & 80.652 & $2.32 E-03$ & 83.977 & $1.57 E-03$ & 84.018 & $1.26 E-03$ & 93.751 & $1.87 E-03$ \\
\hline M1-5 & 147.69 & $2.59 E-03$ & 81.132 & $3.76 E-03$ & 96.077 & $2.00 E-03$ & 88.054 & $3.35 E-03$ & 138.399 & $1.48 E-03$ \\
\hline M1-6 & 144.31 & $2.31 E-03$ & 103.599 & $9.64 E-04$ & 100.549 & $2.08 E-03$ & 83.809 & $1.21 E-03$ & 148.134 & $2.20 E-03$ \\
\hline
\end{tabular}

TABLE 9: Solution time, loops, and RPE of DDM method for model 2.

\begin{tabular}{|c|c|c|c|c|c|c|c|c|c|c|}
\hline \multirow{3}{*}{ Damage scenario } & \multicolumn{10}{|c|}{ Speed parameter } \\
\hline & \multicolumn{2}{|c|}{0.1} & \multicolumn{2}{|c|}{0.3} & \multicolumn{2}{|c|}{0.5} & \multicolumn{2}{|c|}{0.7} & \multicolumn{2}{|c|}{0.9} \\
\hline & $\mathrm{ST}(\mathrm{s})$ & RPE (\%) & $\mathrm{ST}(\mathrm{s})$ & RPE (\%) & ST (s) & RPE (\%) & $\mathrm{ST}(\mathrm{s})$ & RPE (\%) & ST (s) & RPE (\%) \\
\hline M1-1 & 126.055 & $2.15 E-03$ & 109.767 & $3.68 E-03$ & 111.271 & $2.64 E-03$ & 126.776 & $3.42 E-03$ & 110.576 & $2.91 E-03$ \\
\hline M1-2 & 262.790 & $4.16 E-03$ & 237.909 & $6.14 E-03$ & 230.317 & $3.34 E-03$ & 224.621 & $5.16 E-03$ & 212.940 & $6.05 E-03$ \\
\hline M1-3 & 289.739 & $5.89 E-03$ & 318.109 & $4.91 E-03$ & 292.011 & $2.87 E-03$ & 257.903 & $4.57 E-03$ & 298.917 & $4.70 E-03$ \\
\hline M1-4 & 155.150 & $5.73 E-03$ & 165.956 & $5.69 E-03$ & 165.974 & $3.22 E-03$ & 164.765 & $2.82 E-03$ & 166.811 & $4.48 E-03$ \\
\hline M1-5 & 299.442 & $3.55 E-03$ & 252.091 & $5.58 E-03$ & 329.452 & $5.02 E-03$ & 252.018 & $6.32 E-03$ & 270.098 & $3.28 E-03$ \\
\hline M1-6 & 315.672 & $4.24 E-03$ & 219.352 & $2.69 E-03$ & 263.828 & $3.36 E-03$ & 217.605 & $3.24 E-03$ & 232.333 & $6.60 E-03$ \\
\hline
\end{tabular}

\subsubsection{Relative Efficiency Parameter of Adjoint Variable Method} with respect to DDM. Figure 12 shows $\mathrm{REP}_{\mathrm{adj}, \mathrm{ddm}}$ changes with respect to speed parameter in different scenarios.

Table 10 shows that in different scenarios and for different speed parameters, the efficiency parameter is between 1.9713 and 5.5478 for model 1 and 1.5659 and 2.9893 for model 2 and its average is 2.8948 and 2.2248 for models 1 and 2 , respectively. Compared to DDM, the adjoint variable method is more efficient and needs $60.9 \%$ less computational effort.

Changes in the average of $\mathrm{REP}_{\mathrm{adj}, \mathrm{ddm}}$ in different scenarios with speed ratio are shown in Figure 13. As shown in this figure, $\mathrm{REP}_{\mathrm{adj}, \mathrm{ddm}}$ increases with speed ratios lightly. This indicates that efficiency of $\mathrm{ADM}$ with respect to $\mathrm{DDM}$ increases with speed ratio.

\subsubsection{Relative Efficiency Parameter of Adjoint Variable Method} with respect to Approximation and Semianalytical Methods. Figure 14 shows $\mathrm{REP}_{\mathrm{adj}, \mathrm{FD}}$ and $\mathrm{REP}_{\mathrm{adj}, \mathrm{DDMFD}}$ changes with respect to speed parameter in different scenarios.

Table 11 shows the relative efficiency parameter for different speed parameters in different scenarios. It can be seen that the efficiency parameter is between 31.93 and 783.07 for finite difference method and 49.16 and 505.42 for DDMFD method with the average of 167.14 and 145.69 for FD and DDMFD methods, respectively. Therefore the adjoint variable method is extremely successful and the computational cost of this method is just $0.6 \%$ and $0.69 \%$ of FD and DDMFD methods, respectively.

Changes in the average of $\mathrm{REP}_{\mathrm{adj}, \mathrm{fd} \& \mathrm{ddmfd}}$ in different scenarios with speed ratio are shown in Figure 15. As shown in this figure, $\mathrm{REP}_{\mathrm{adj}, \mathrm{fd} \& \mathrm{ddmfd}}$ rapidly increases with speed ratio, which indicates the superiority of ADM over FD and DDMFD methods increases with speed ratio.
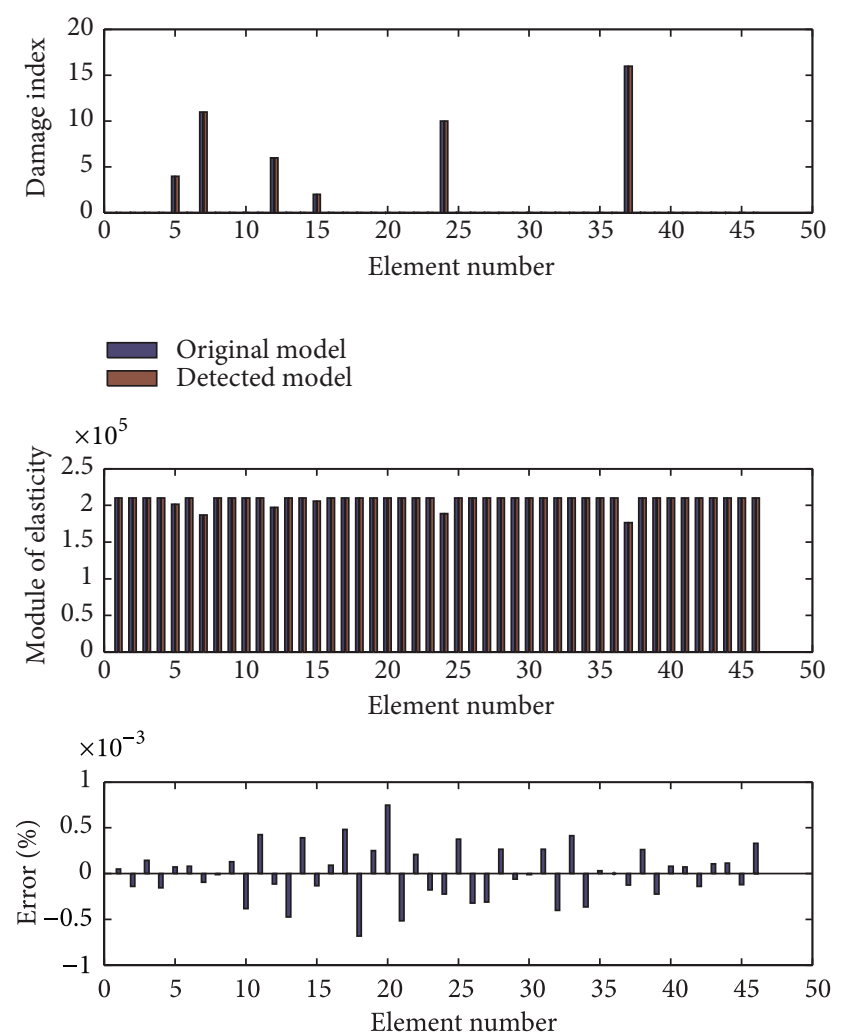

FIGURE 6: Detection of damage location and amount in elements 5, $7,12,15,24$, and 37 and distribution of error in different elements with ADM scheme.

5.4.3. Relative Efficiency Parameter of DDMFD with respect to FD Method. Figure 16 shows $\mathrm{REP}_{\text {ddmfd,fd }}$ changes with respect to speed parameter in different scenarios. 
TABLE 10: $\mathrm{REP}_{\text {adj,ddm }}$ ranges in different scenarios.

\begin{tabular}{|c|c|c|c|c|c|c|}
\hline \multirow{2}{*}{ Damage scenario } & \multicolumn{2}{|c|}{ Max REP } & \multicolumn{2}{|c|}{ Min REP } & \multicolumn{2}{|c|}{ Average } \\
\hline & Model 1 & Model 2 & Model 1 & Model 2 & Model 1 & Model 2 \\
\hline M1-1 & 4.2291 & 1.9262 & 2.3373 & 1.5659 & 2.8785 & 1.7420 \\
\hline M1-2 & 3.8674 & 2.9893 & 2.3880 & 2.3178 & 3.1318 & 2.6008 \\
\hline M1-3 & 3.5062 & 2.8052 & 2.6852 & 2.0291 & 3.0292 & 2.4267 \\
\hline M1-4 & 3.6137 & 2.2448 & 1.9713 & 2.0117 & 2.5682 & 2.0893 \\
\hline M1-5 & 3.5047 & 2.9353 & 2.1168 & 1.6647 & 2.5290 & 2.2304 \\
\hline M1-6 & 5.5478 & 2.6344 & 2.2784 & 2.0035 & 3.2318 & 2.2587 \\
\hline Total & 5.5478 & 2.9893 & 1.9713 & 1.5659 & 2.8948 & 2.2248 \\
\hline
\end{tabular}

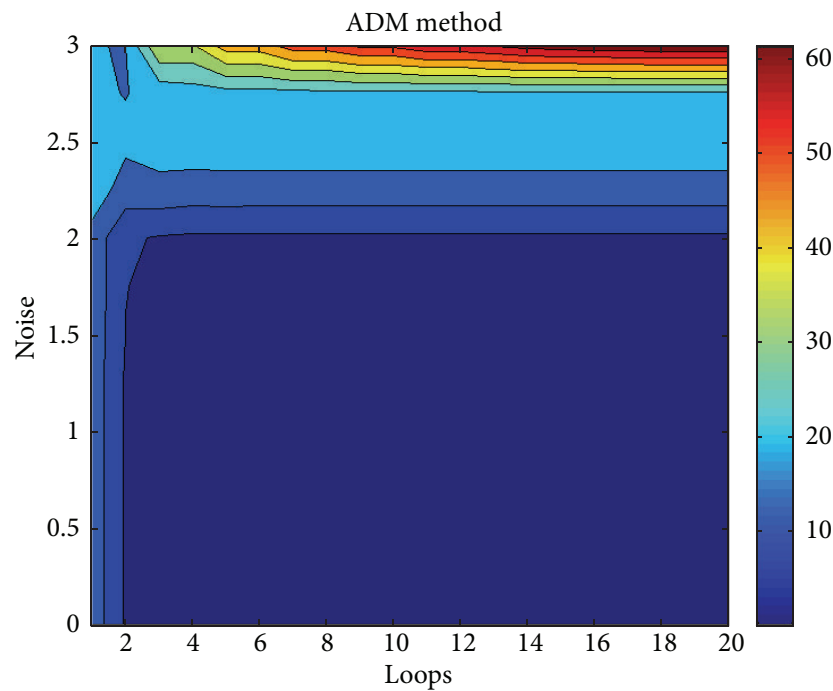

(a) ADM method

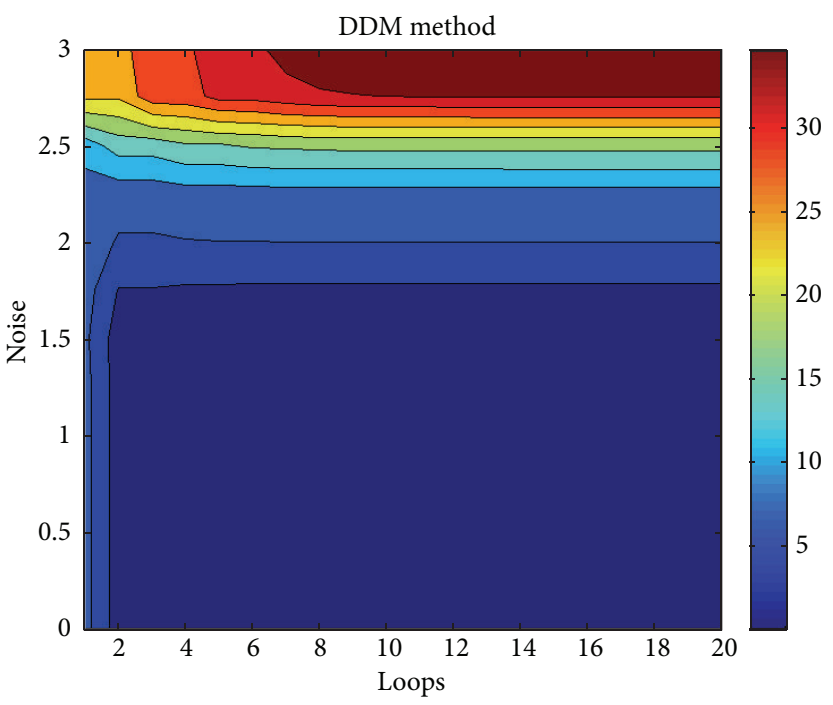

(b) DDM method

FIGURE 7: RPE contours with respect to noise level and loops in model 2.

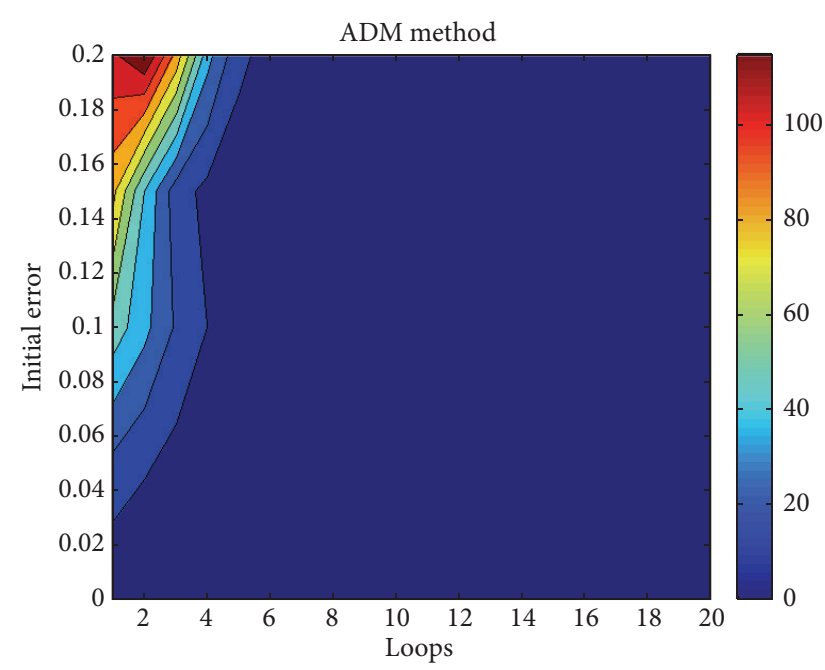

(a) ADM method

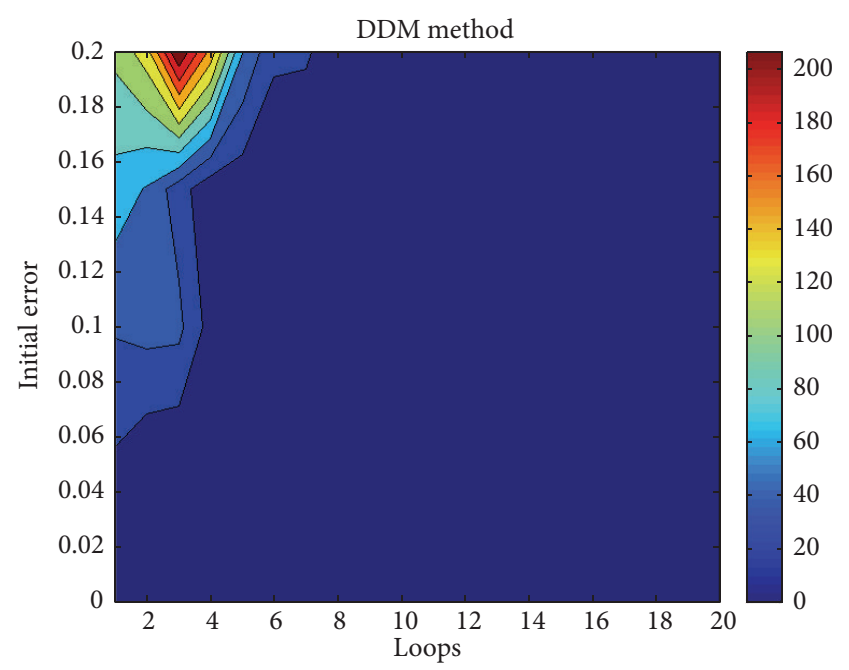

(b) DDM method

FIGURE 8: RPE contours with respect to initial error and loops in model 2. 


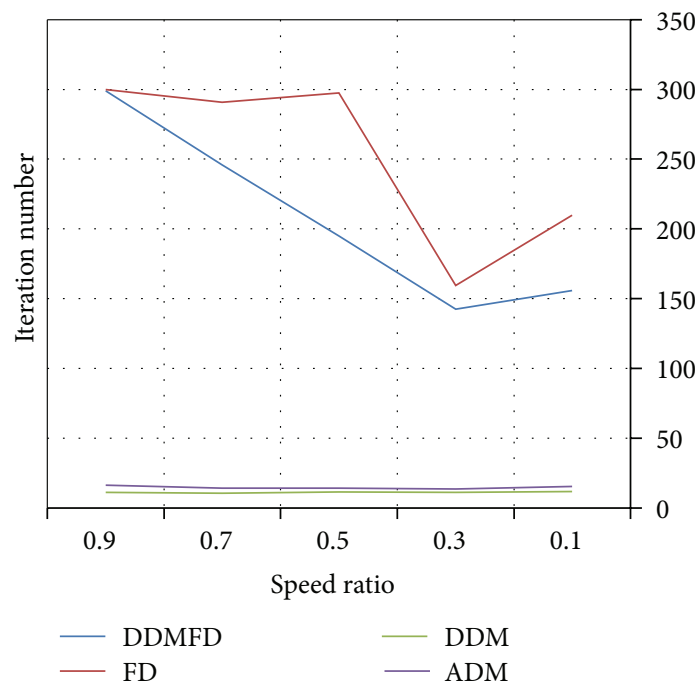

(a) All methods

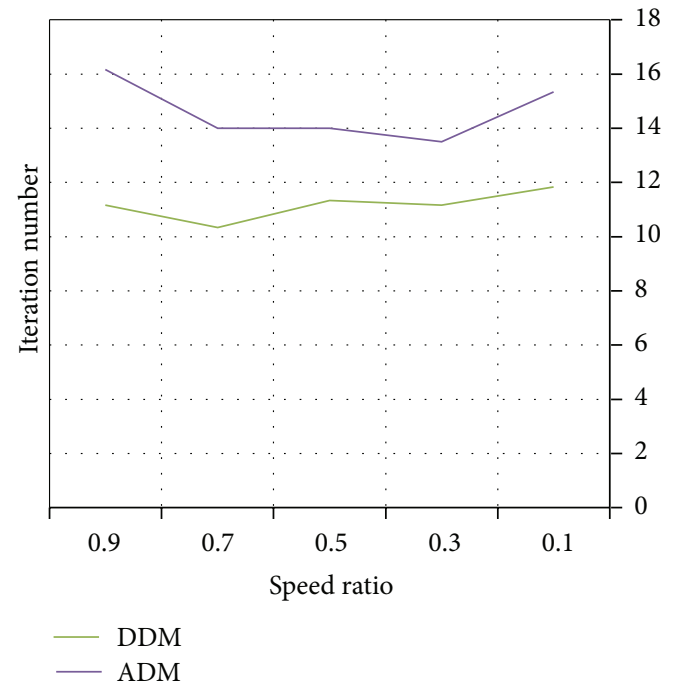

(b) Analytical discrete methods

FIGURE 9: Comparison of iteration number between different sensitivity methods.

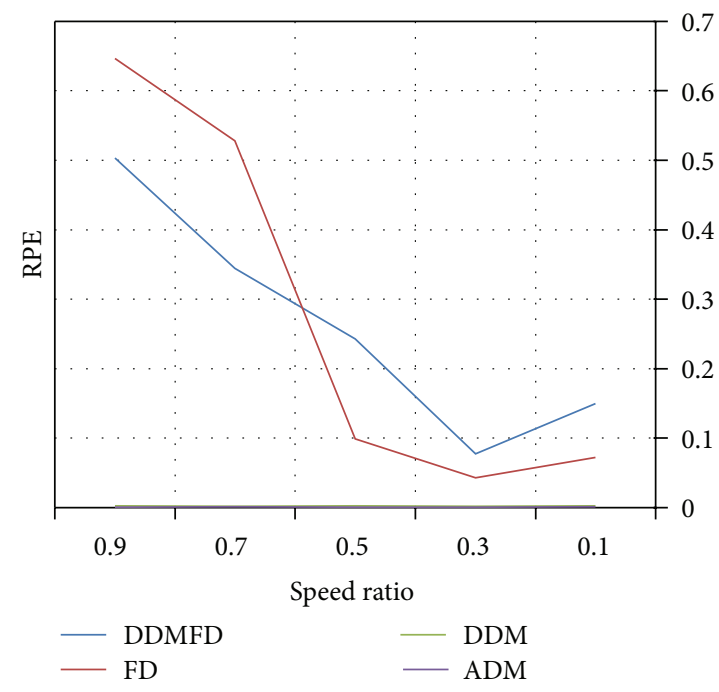

(a) All methods

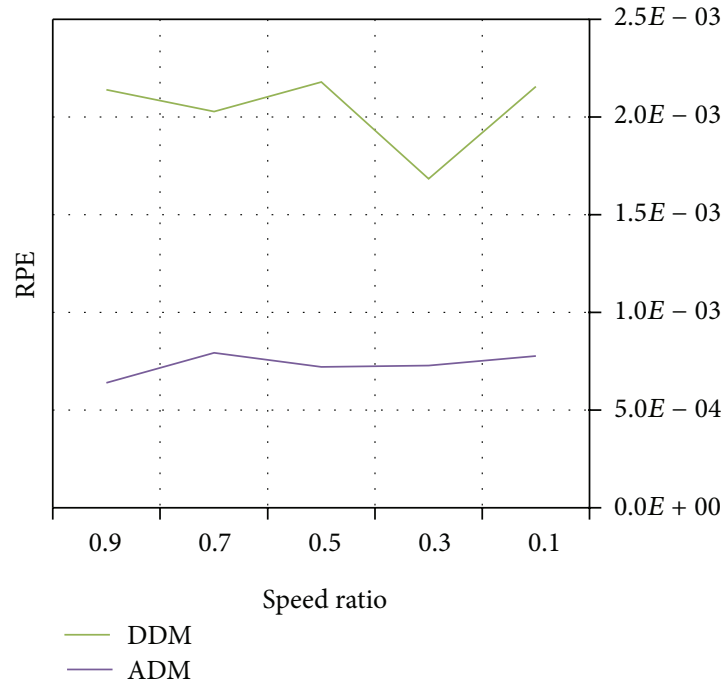

(b) Analytical discrete methods

FIGURE 10: Comparison of RPE between different sensitivity methods.

Table 12 shows the relative efficiency parameter for different speed parameters in different scenarios. Again, the efficiency parameter is between 0.343 and 1.726 with the average of 1.044. Thus, compared to FD method, the DDMFD method is slightly more effective.

Changes in the average of $\mathrm{REP}_{\mathrm{ddmfd}, \mathrm{fd}}$ in different scenarios with speed ratio are shown in Figure 17. As shown in this figure, $\mathrm{REP}_{\mathrm{ddmfd}, \mathrm{fd}}$ increases with speed ratio. For speed ratio lower than 0.5 efficiency parameter is lower than 1 which means FD method is more effective for this range.

5.5. Stability. Figures 3 and 7 show that all described methods are sensitive to noise and if noise level exceeds the values stated in Table 11, these methods lose their efficiency and are not able to detect damage properly. Hence, in cases with noise level larger than the values stated in Table 13, a denoising tool like wavelet transforms should be used along with the sensitivity methods. Wavelet transforms are mainly attractive because of their ability to compress and encode information to reduce noise or to detect any local singular behavior of a signal [37].

Stability comparison of different methods shows that FD is the most stable method and stability of analytical methods is slightly lower than that.

Figures 4 and 8 show stability of sensitivity methods with respect to the initial error. Summary of results for stability investigation is presented in Table 13, which suggests that stability of numerical methods is better than analytical methods. 


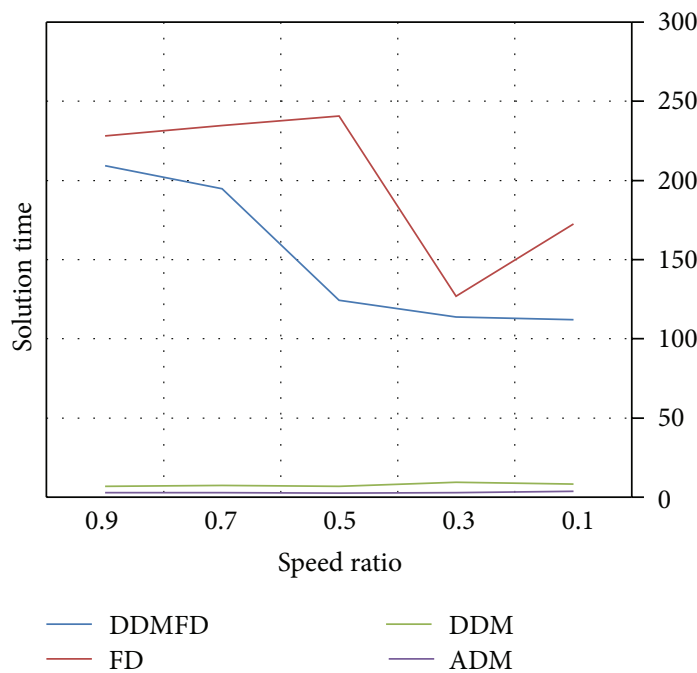

(a) All methods

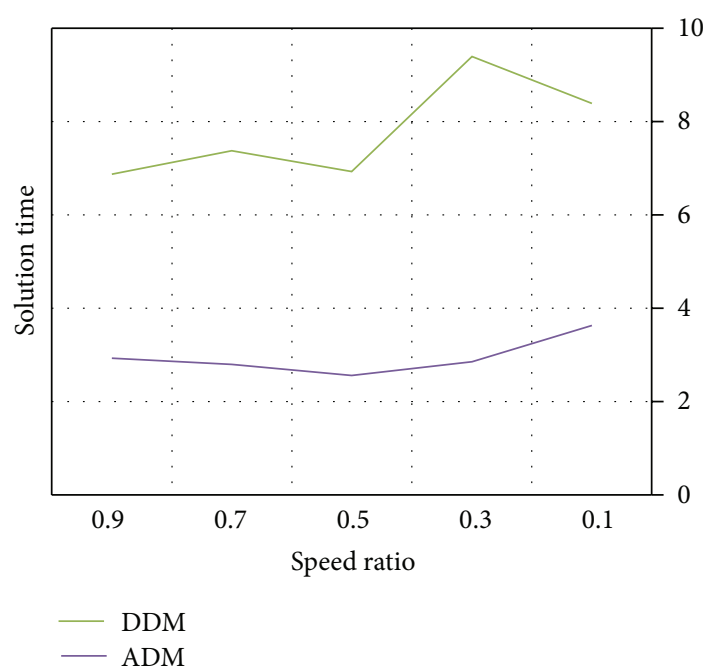

(b) Analytical discrete methods

FIGURE 11: Comparison of solution time between different sensitivity methods.

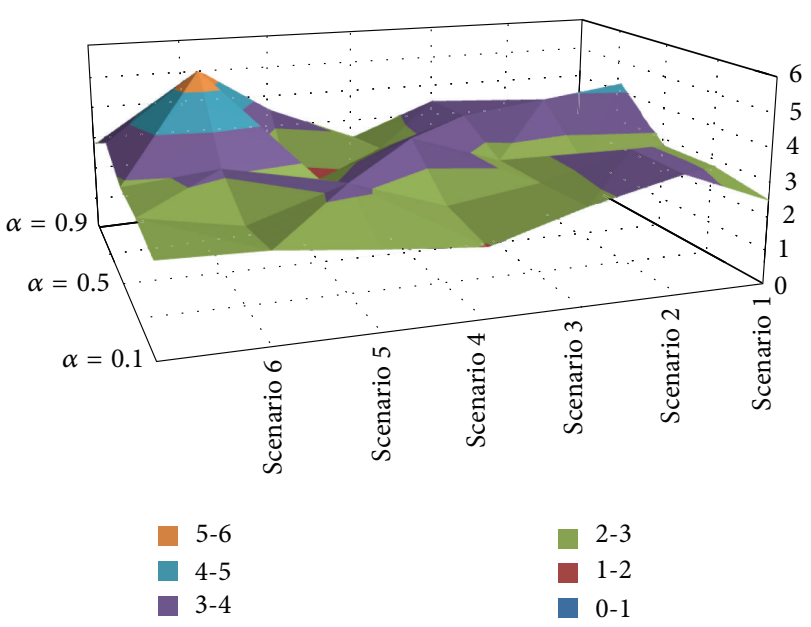

(a) Model 1

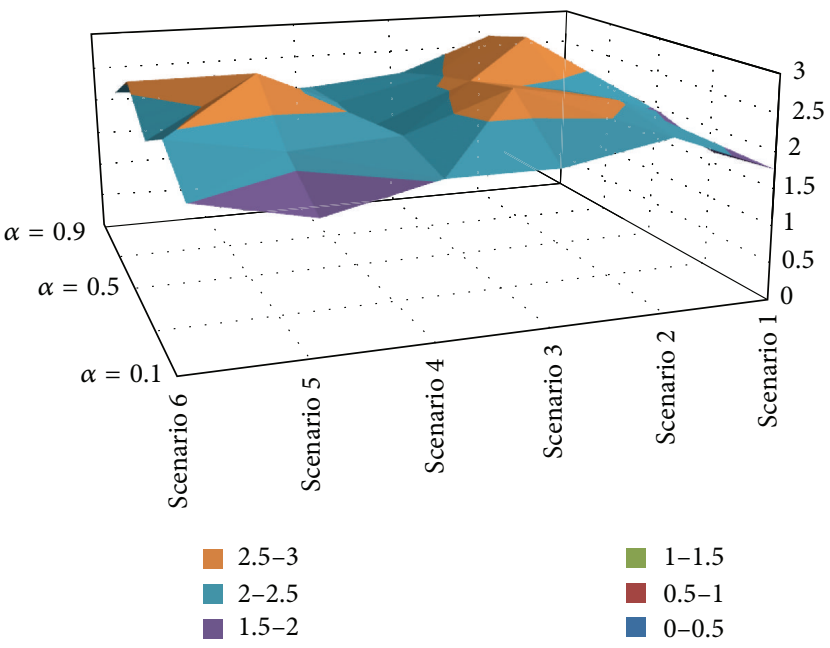

(b) Model 2

FIGURE 12: $\mathrm{REP}_{\mathrm{adj}, \mathrm{ddm}}$ changes in different scenarios with respect to speed parameter.

TABLE 11: $\mathrm{REP}_{\text {adjadm }}$ ranges in different scenarios.

\begin{tabular}{lcccccr}
\hline \multirow{2}{*}{ Damage scenario } & \multicolumn{2}{c}{ Max REP } & \multicolumn{2}{c}{ Min REP } & \multicolumn{2}{c}{ Average } \\
& FD & DDMFD & FD & DDMFD & FD & 156.94 \\
M1-1 & 295.79 & 268.06 & 40.073 & 51.54 & 76.39 & 200.82 \\
M1-2 & 478.80 & 307.33 & 53.87 & 63.32 & 142.48 & 183.35 \\
M1-3 & 291.43 & 241.65 & 33.28 & 49.16 & 146.99 & 148.26 \\
M1-4 & 298.07 & 174.74 & 43.55 & 56.07 & 105.03 & 114.62 \\
M1-5 & 226.85 & 276.31 & 41.57 & 65.47 & 250.56 & 187.09 \\
M1-6 & 783.07 & 505.42 & 31.93 & 49.16 & 167.14 & 145.69 \\
\hline Total & 783.07 & 505.42 & 31.93 & & & 163 \\
\hline
\end{tabular}




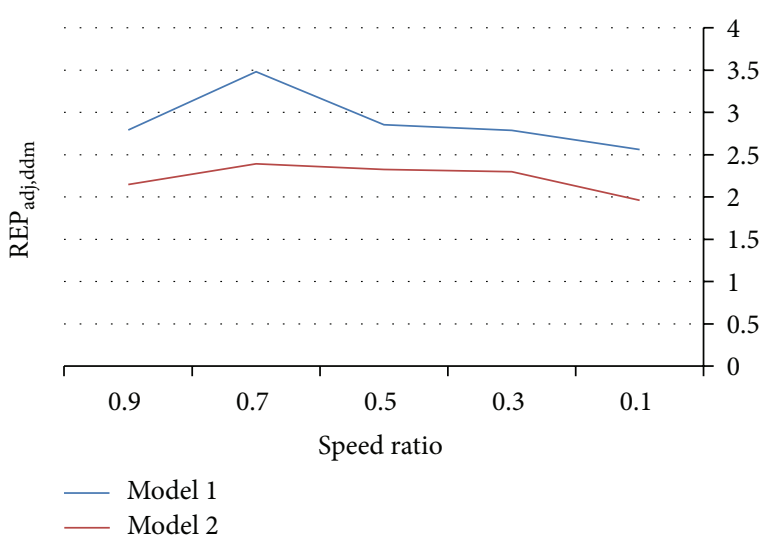

FIGURE 13: Average of $\mathrm{REP}_{\text {adj,ddm }}$ changes with respect to speed parameter.

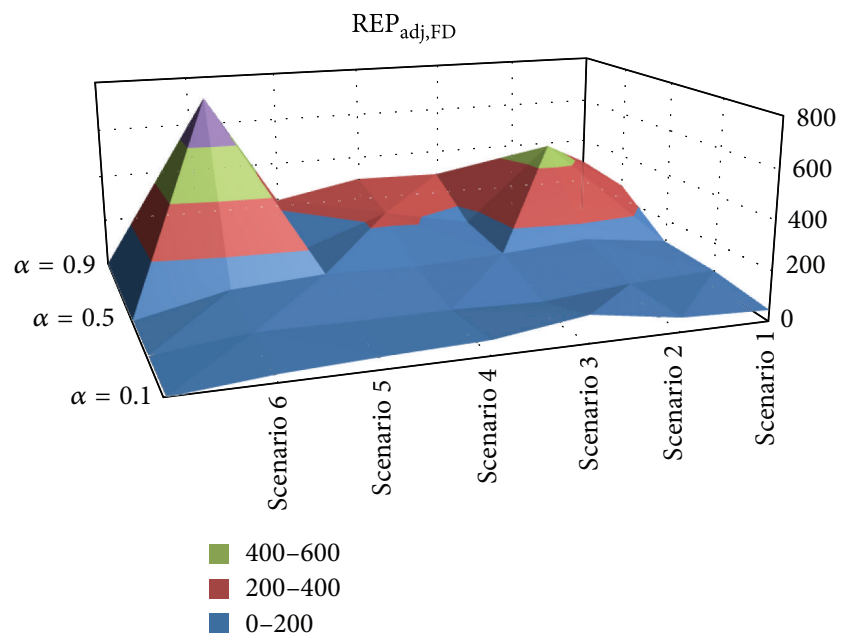

(a)

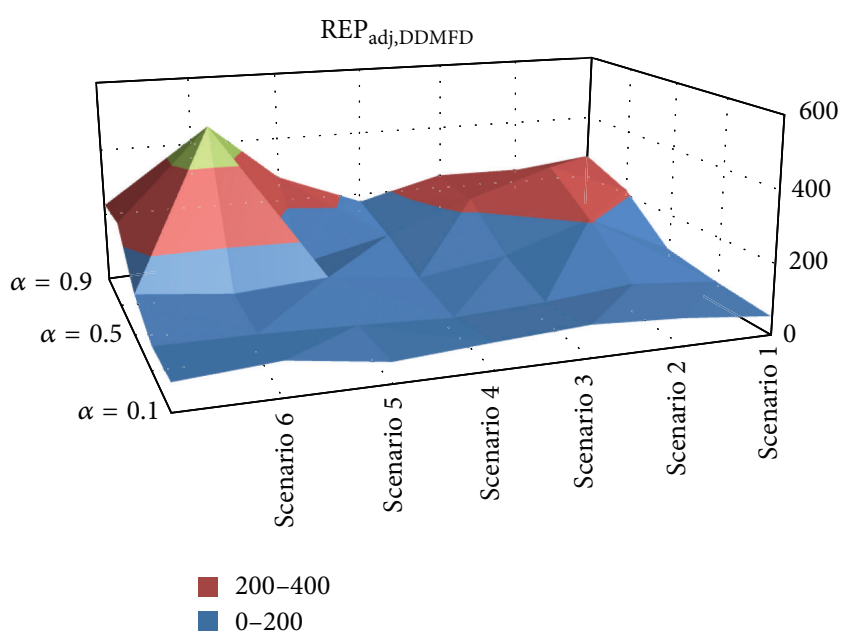

(b)

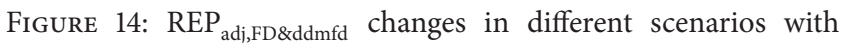
respect to speed parameter.

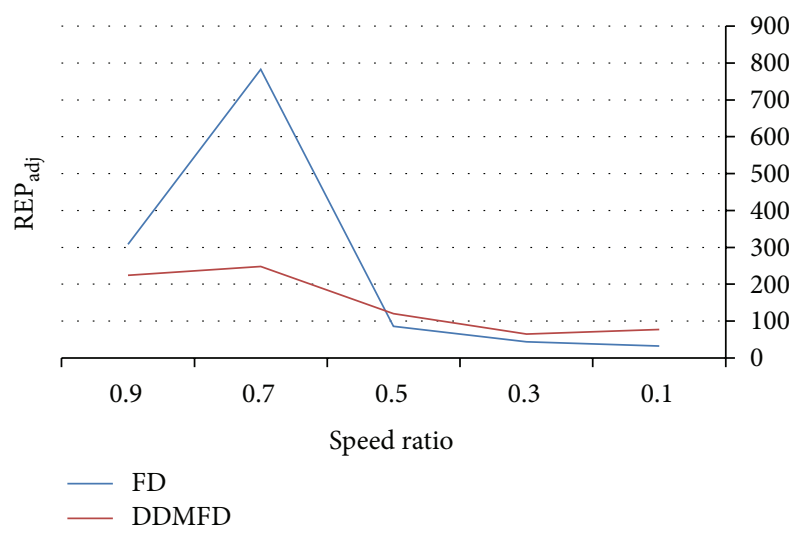

FIGURE 15: Average of $\mathrm{REP}_{\text {adj,fd\&ddmfd }}$ changes with respect to speed parameter.

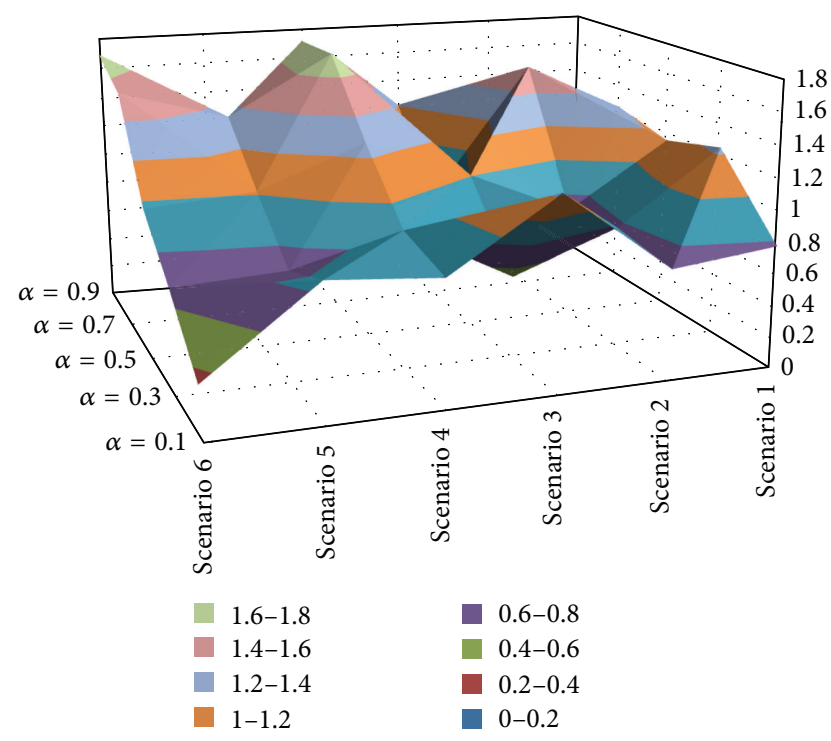

FIGURE 16: $\mathrm{REP}_{\mathrm{ddmfd} \text {,fd }}$ changes in different scenarios with respect to speed parameter.

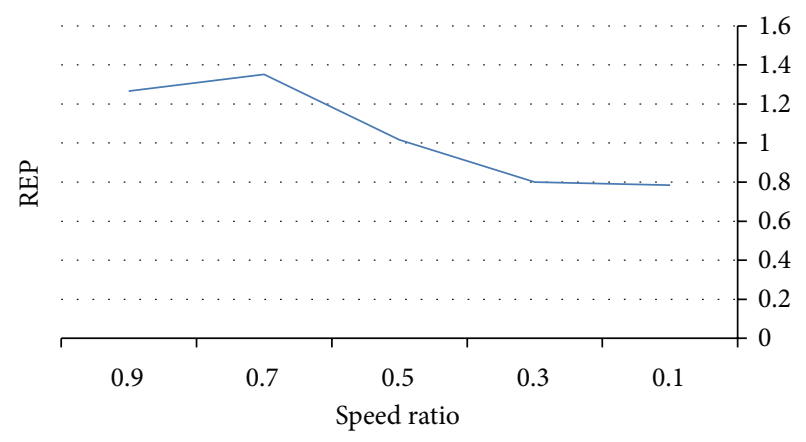

DDMFD/FD

FIGURE 17: Average of $\mathrm{REP}_{\mathrm{ddmfd}, \mathrm{fd}}$ changes with respect to speed parameter. 
TABLE 12: $\mathrm{REP}_{\mathrm{adj}, \mathrm{adm}}$ ranges in different scenarios.

\begin{tabular}{lccc}
\hline Damage scenario & Max REP & Min REP & Average \\
\hline M1-1 & 1.23321 & 0.777484 & 1.095359 \\
M1-2 & 1.557902 & 0.705181 & 0.964746 \\
M1-3 & 1.226306 & 0.52565 & 0.931323 \\
M1-4 & 1.726018 & 0.796804 & 1.277344 \\
M1-5 & 1.349058 & 0.706861 & 0.949126 \\
M1-6 & 1.686819 & 0.343253 & 1.04332 \\
\hline Total & 1.726018 & 0.343253 & 1.043536 \\
\hline
\end{tabular}

TABLE 13: Stability of different methods against noise and initial error.

\begin{tabular}{lcccc}
\hline \multirow{2}{*}{ Method } & \multicolumn{2}{c}{ Noise level } & \multicolumn{2}{c}{ Initial error } \\
& Model 1 & Model 2 & Model 1 & Model 2 \\
\hline ADM & $2.5 \%$ & $2.4 \%$ & $20 \%$ & $20 \%$ \\
DDM & $2.5 \%$ & $2.3 \%$ & $20 \%$ & $20 \%$ \\
FD & $2.7 \%$ & - & $30 \%$ & - \\
DDMFD & $2.4 \%$ & - & $30 \%$ & - \\
\hline
\end{tabular}

Comparing Figures 3 and 4 shows that divergence type due to initial error is sharp unlike the noise level which occurs gradually.

\section{Conclusion}

Different sensitivity-based damage detection methods are presented and acceleration time history data affected by a moving vehicle with specified load is used for damage detection procedure. Newmark method is used to calculate the structural dynamic response and its dynamic response sensitivities are calculated by four different sensitivity methods (finite difference method (FD), semianalytical discrete method (DDMFD), direct differential method (DDM), and adjoint variable method (ADM)).

Different damaged structures including single, multiple, and random damage are considered and efficiency of four aforementioned sensitivity methods is compared and following remarks are made.

(i) The advantage of the finite difference method is obvious. If structural analysis can be performed and the performance measure can be obtained as a result of structural analysis, then FD method is independent of the problem types considered. However, sensitivity computation costs become the main concern in the damage detection process for the large problems. Comparison of sensitivity methods shows that FD method is the most expensive method among other procedures.

(ii) Semianalytical discrete method (DDMFD) alleviates some disadvantages of FD method and its computational cost is rather lower than FD method.

(iii) The major disadvantage of the finite difference based methods (FD and DDMFD) is the accuracy of their sensitivity results. Depending on perturbation size, sensitivity results are quite different. As a result, it is very difficult to determine design perturbation sizes that work for all problems.

(iv) The computational cost of damage detection procedure using these methods is too expensive and these methods are infeasible for large-scale problems containing many variables, for example, the second case study (model 2).

(v) The DDM is an accurate and efficient method to compute sensitivity matrix. This method directly solves for the design dependency of a state variable and then computes performance sensitivity using the chain rule of differentiation. The comparative study shows this has a very low computational cost method in all cases and is more accurate than finite difference methods.

(vi) ADM calculates each element of sensitivity matrix separately by defining an adjoint variable parameter. The main advantage is in evaluating the dynamic response; an analytical solution exists which significantly increases the speed and accuracy of the solution. The comparative study shows that efficiency parameter of ADM is 2.89, 167.14, and 145.69 compared to DDM, FD, and DDMFD methods, respectively. This result indicates that ADM is extremely successful and can be applied as a powerful tool in SHM.

(vii) Investigations of initial assumption error in stability of methods show that finite difference based methods have more enhanced stability than analytical discrete methods and all sensitivity-based methods have moderate stability against initial assumption error.

(viii) The drawback of all sensitivity-based methods is their low stability against input measurement noise (about $2.5 \%$ ), which can be improved by using low-pass denoising tools.

\section{Conflict of Interests}

The authors declare that there is no conflict of interests regarding the publication of this paper.

\section{References}

[1] H. Wenzel, Health Monitoring of Bridges, John Wiley \& Sons, Chichester, UK, 2009.

[2] P. M. Pawar and R. Ganguli, Structural Health Monitoring Using Genetic Fuzzy Systems, Springer, London, UK, 2011.

[3] S. Gopalakrishnan, M. Ruzzene, and S. Hanagud, Computational Techniques for Structural Health Monitoring, Springer, London, UK, 2011.

[4] A. Morrasi and F. Vestroni, Dynamic Methods for Damage Detection in Structures, Springer, New York, NY, USA, 2008.

[5] Z. R. Lu and J. K. Liu, "Parameters identification for a coupled bridge-vehicle system with spring-mass attachments," Applied Mathematics and Computation, vol. 219, no. 17, pp. 9174-9186, 2013. 
[6] S. W. Doebling, C. R. Farrar, and M. B. Prime, "A summary review of vibration-based damage identification methods," Shock and Vibration Digest, vol. 30, no. 2, pp. 91-105, 1998.

[7] S. W. Doebling, C. R. Farrar, M. B. Prime, and D. W. Shevitz, "Damage identification and health monitoring of structural and mechanical systems from changes in their vibration characteristics; a literature review," Tech. Rep. LA-13070- MS,UC-900, Los Alamos National Laboratory, Los Alamos, NM, USA, 1996.

[8] O. S. Salawu, "Detection of structural damage through changes in frequency: a review," Engineering Structures, vol. 19, no. 9, pp. 718-723, 1997.

[9] Y. Zou, L. Tong, and G. P. Steven, "Vibration-based modeldependent damage (delamination) identification and health monitoring for composite structures-a review," Journal of Sound and Vibration, vol. 230, no. 2, pp. 357-378, 2000.

[10] S. Alampalli and G. Fu, "Remote monitoring systems for bridge condition," Client Report 94, Transportation Research and Development Bureau, New York State Department of Transportation, New York, NY, USA, 1994.

[11] S. Alampalli, G. Fu, and E. W. Dillon, "Measuring bridge vibration for detection of structural damage," Research Report 165, Transportation Researchand Development Bureau, New York State Department of Transportation, 1995.

[12] J. R. Casas and A. C. Aparicio, "Structural damage identification from dynamic-test data," Journal of Structural Engineering, American Society of Chemical Engineers, vol. 120, no. 8, pp. 24372449, 1994.

[13] P. Cawley and R. D. Adams, "The location of defects in structures from measurements of natural frequencies," The Journal of Strain Analysis for Engineering Design, vol. 14, no. 2, pp. 49-57, 1979.

[14] M. I. Friswell, J. E. T. Penny, and D. A. L. Wilson, "Using vibration data and statistical measures to locate damage in structures," Modal Analysis, vol. 9, no. 4, pp. 239-254, 1994.

[15] Y. Narkis, "Identification of crack location in vibrating simply supported beams," Journal of Sound and Vibration, vol. 172, no. 4, pp. 549-558, 1994.

[16] A. K. Pandey, M. Biswas, and M. M. Samman, "Damage detection from changes in curvature mode shapes," Journal of Sound and Vibration, vol. 145, no. 2, pp. 321-332, 1991.

[17] C. P. Ratcliffe, "Damage detection using a modified laplacian operator on mode shape data," Journal of Sound and Vibration, vol. 204, no. 3, pp. 505-517, 1997.

[18] P. F. Rizos, N. Aspragathos, and A. D. Dimarogonas, "Identification of crack location and magnitude in a cantilever beam from the vibration modes," Journal of Sound and Vibration, vol. 138, no. 3, pp. 381-388, 1990.

[19] A. K. Pandey and M. Biswas, "Damage detection in structures using changes in flexibility," Journal of Sound and Vibration, vol. 169, no. 1, pp. 3-17, 1994.

[20] T. W. Lim, "Structural damage detection using modal test data," AIAA Journal, vol. 29, no. 12, pp. 2271-2274, 1991.

[21] D. Wu and S. S. Law, "Model error correction from truncated modal flexibility sensitivity and generic parameters: part Isimulation," Mechanical Systems and Signal Processing, vol. 18, no. 6, pp. 1381-1399, 2004.

[22] K. F. Alvin, A. N. Robertson, G. W. Reich, and K. C. Park, "Structural system identification: from reality to models," Computers and Structures, vol. 81, no. 12, pp. 1149-1176, 2003.

[23] R. Sieniawska, P. Śniady, and S. Zukowski, "Identification of the structure parameters applying a moving load," Journal of Sound and Vibration, vol. 319, no. 1-2, pp. 355-365, 2009.
[24] C. Gentile and A. Saisi, "Ambient vibration testing of historic masonry towers for structural identification and damage assessment," Construction and Building Materials, vol. 21, no. 6, pp. 1311-1321, 2007.

[25] W. X. Ren and Z. H. Zong, "Output-only modal parameter identification of civil engineering structures," Structural Engineering and Mechanics, vol. 17, no. 3-4, pp. 429-444, 2004.

[26] S. S. Law, J. Q. Bu, X. Q. Zhu, and S. L. Chan, "Vehicle axle loads identification using finite element method," Journal of Engineering Structures, vol. 26, no. 8, pp. 1143-1153, 2004.

[27] R. J. Jiang, F. T. K. Au, and Y. K. Cheung, "Identification of vehicles moving on continuous bridges with rough surface," Journal of Sound and Vibration, vol. 274, no. 3-5, pp. 1045-1063, 2004.

[28] X. Q. Zhu and S. S. Law, "Damage detection in simply supported concrete bridge structure under moving vehicular loads," Journal of Vibration and Acoustics, vol. 129, no. 1, pp. 58-65, 2007.

[29] M. I. Friswell and J. E. Mottershead, Finite Element Model Updating in Structural Dynamics, vol. 38 of Solid Mechanics and its Applications, Kluwer Academic Publishers Group, Dordrecht, The Netherlands, 1995.

[30] X. Q. Zhu and H. Hao, Damage Detection of Bridge Beam Structures under Moving Loads, School of Civil and Resource Engineering, University of Western Australia, 2007.

[31] T. Lauwagie, H. Sol, and E. Dascotte, Damage Identification in Beams Using Inverse Methods, Department of Mechanical Engineering, Katholieke Universiteit Leuven, Leuven, Belgium, 2002.

[32] D. Cacuci, Sensitivity and Uncertainty Analysis, Volume 1, Chapman \& Hall/CRC, Boca Raton, Fla, USA, 2003.

[33] K. K. Choi and N. H. Kim, Structural Sensitivity Analysis and Optimization 1: Linear Systems, Springer, New York, NY, USA, 2005.

[34] A. Mirzaee, M. A. Shayanfar, and R. Abbasnia, "Damage detection of bridges using vibration data by adjoint variable method," Shock and Vibration, vol. 2014, Article ID 698658, 17 pages, 2014.

[35] M. Friswell, J. Mottershead, and H. Ahmadian, "Finite-element model updating using experimental test data: parametrization and regularization," Philosophical Transactions of the Royal Society A, vol. 365, pp. 393-410, 2007.

[36] X. Y. Li and S. S. Law, "Adaptive Tikhonov regularization for damage detection based on nonlinear model updating," Mechanical Systems and Signal Processing, vol. 24, no. 6, pp. 1646-1664, 2010.

[37] M. Solís, M. Algaba, and P. Galvín, "Continuous wavelet analysis of mode shapes differences for damage detection," Journal of Mechanical Systems and Signal Processing, vol. 40, no. 2, pp. 645666, 2013. 

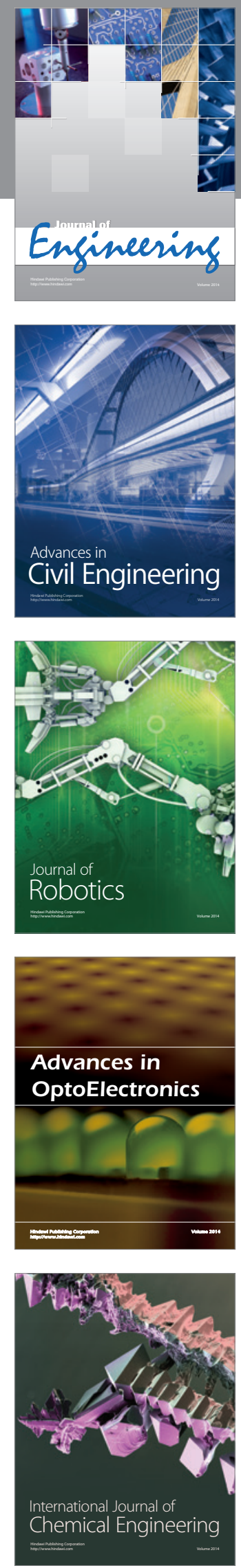

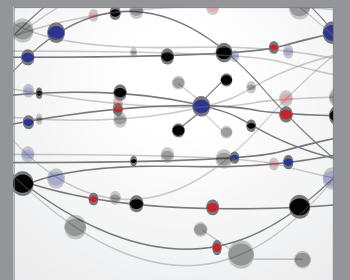

The Scientific World Journal
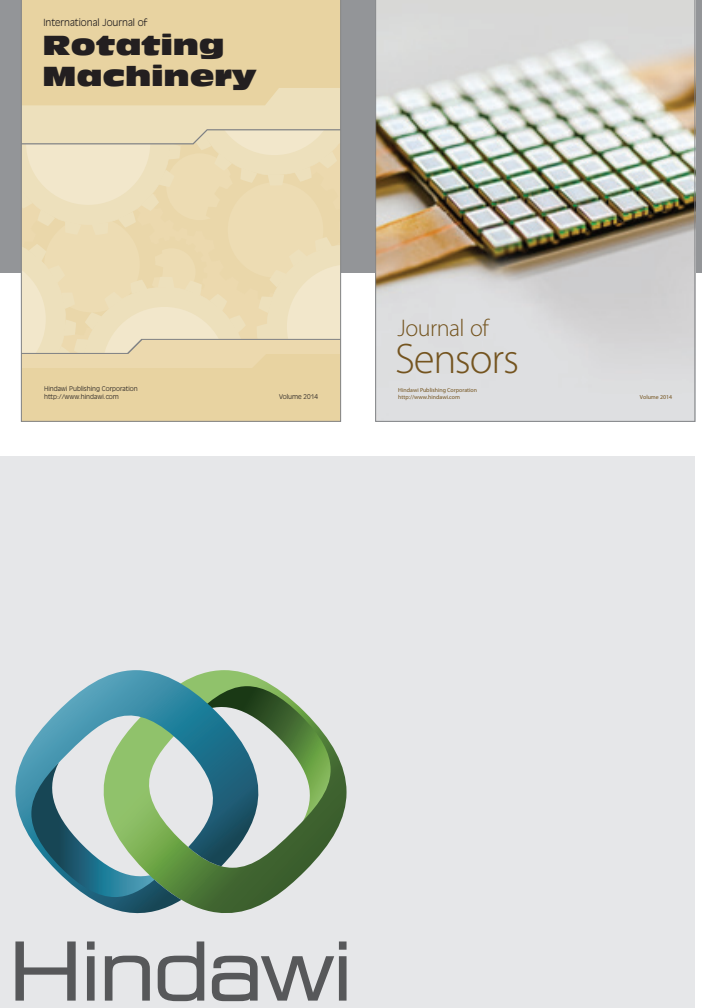

Submit your manuscripts at http://www.hindawi.com
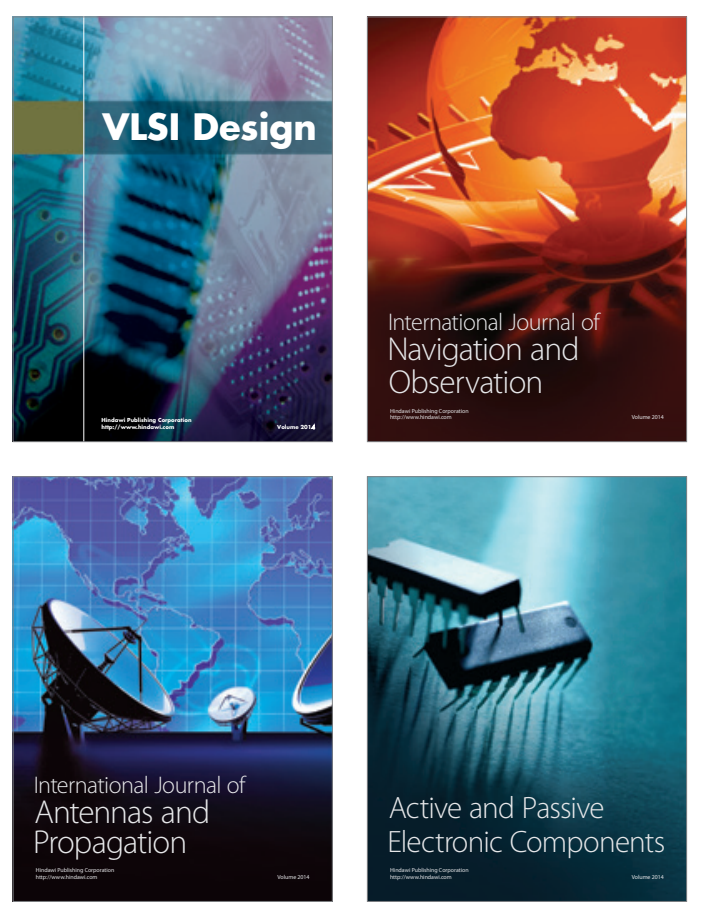
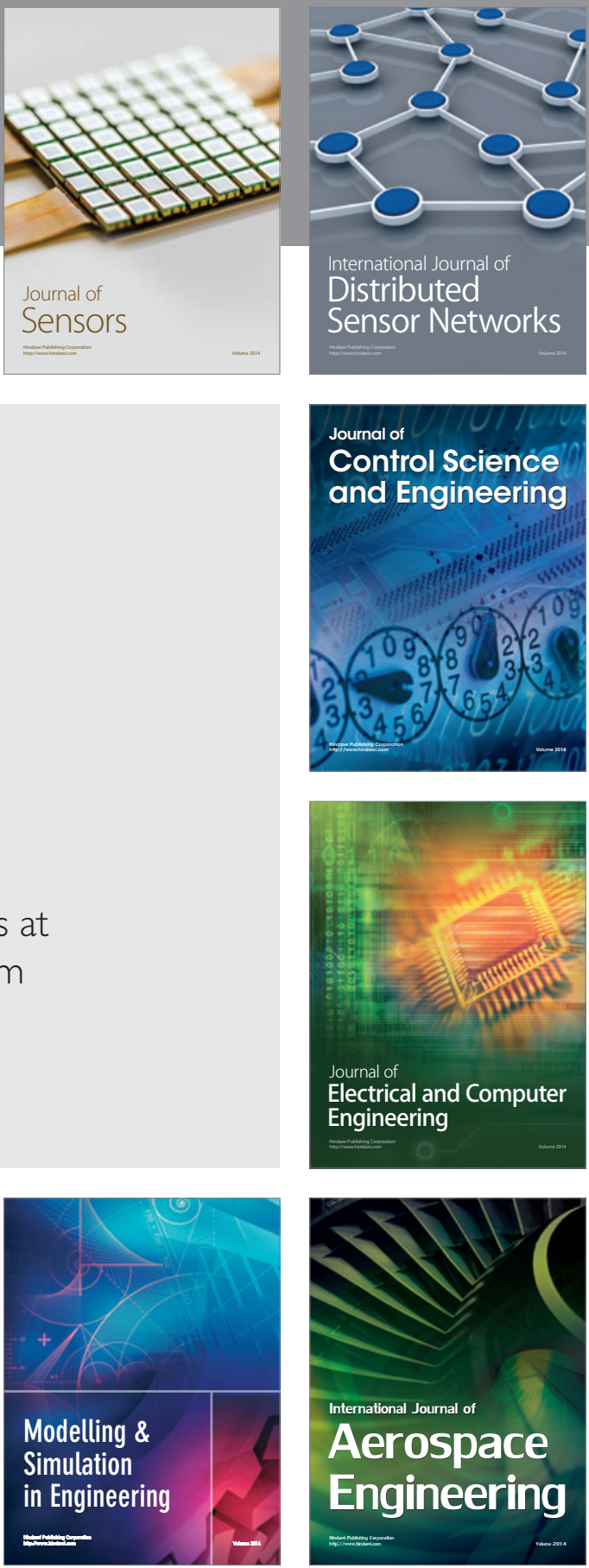

Journal of

Control Science

and Engineering
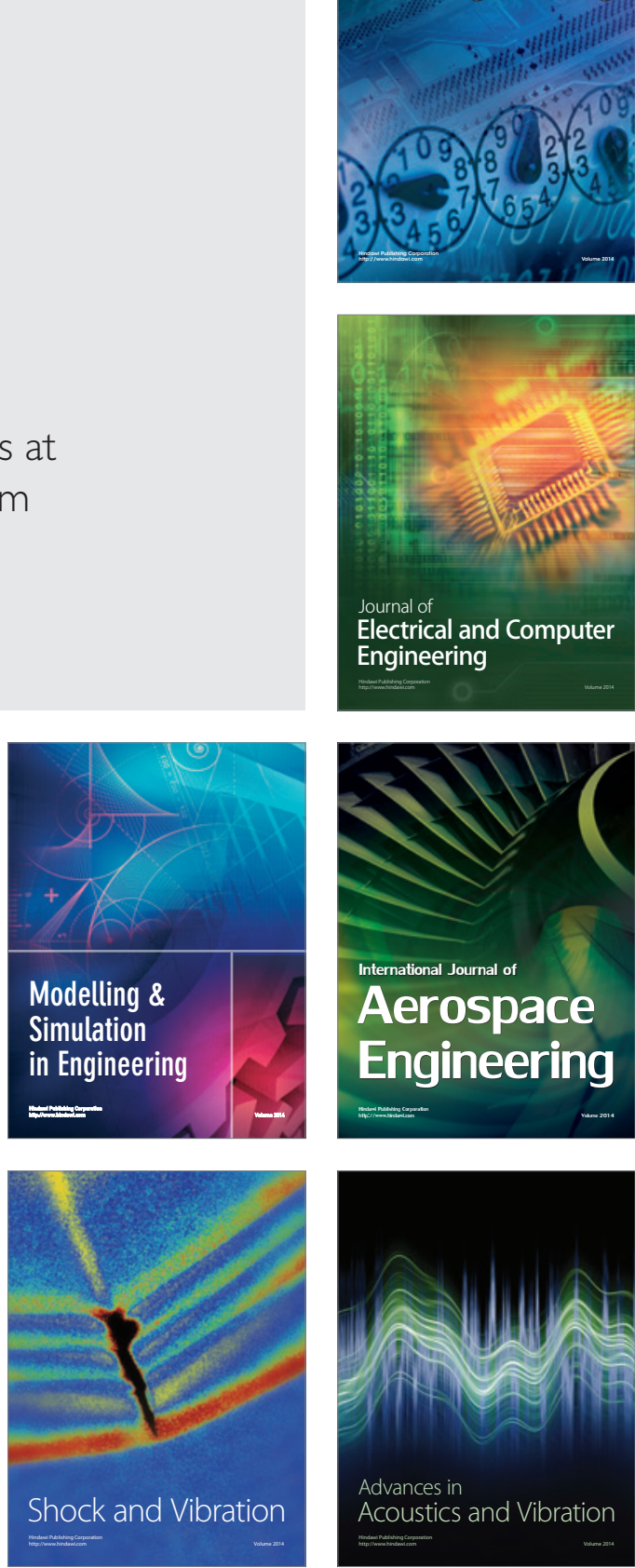